\title{
A More General Model of Price Complexity*
}

\author{
Ioana Chioveanu ${ }^{\dagger}$
}

November 2019

\begin{abstract}
This paper analyses a model of competition where the firms set not only prices but also the complexity levels of their prices (which determine how difficult it is for consumers to assess the price offers). Unlike previous work, in this model, the firms' confusion technology may be nonlinear in the aggregate complexity level. The equilibrium probability of using high complexity increases in the number of firms but decreases in the convexity of the confusion technology. In large markets, the firms use high complexity almost surely. However, the industry profit converges to the highest level with concave technologies and to the lowest level with convex technologies. An increase in consumer sophistication, which benefits the consumers, may not reduce market complexity.
\end{abstract}

Keywords: price complexity, confusion technology, concavity/convexity, oligopoly markets JEL classification: D03, D43, L13

${ }^{*}$ I thank the Editor, Julian Wright, and two anonymous referees for their valuable comments, which significantly improved the paper. I am also grateful to Uğur Akgün, Mark Armstrong, John Bennett, Antonio Cabrales, Tobias Gamp, Yiquan Gu, Matthew Rablen, Antonio Navas, Ran Spiegler, Tobias Wenzel, and audiences at the University of Sheffield (2019) and at the meetings of the European Economic Association (Cologne, 2018), the European Association for Research in Industrial Economics (Athens, 2018), the Association of Southern-European Economic Theorists (Florence, 2018), and the Catalan Economic Society (Barcelona, 2019) for useful comments and suggestions.

${ }^{\dagger}$ Department of Economics and Finance, Brunel University London, Uxbridge, UB8 3PH, UK. Email: ioana.chioveanu@brunel.ac.uk. 


\section{Introduction}

In many markets, including banking, finance, and energy retail, firms' use of partitioned prices, differentiated price formats, or technical language in their price disclosures makes it harder for consumers to assess prices and identify the best offer. Recent research associates price complexity with consumer inertia and lack of sophistication, price and format dispersion, positive mark-ups, and unintended responses to intensified competition. It also raises concerns about its strategic use by firms to create consumer confusion and soften competition.

Discussing the challenges of consumer financial regulation, Campbell (2016) points out that "financial ignorance is pervasive and unsurprising given the complexity of modern financial products". The 2015 UK Competition and Market Authority investigation of the retail banking market found that price complexity may prevent consumers from receiving good value and identifying the best deals. A 2007 EC study of EU mortgage credit markets and the 2011 report by the UK Independent Commission on Banking echo these concerns. ${ }^{1}$

Related theoretical work sends a consistent message that, in homogeneous product markets, the positive effects of stronger competition are weakened by increases in complexity and equilibrium obfuscation, and identifies the possibility that an increase in the number of firms harms consumer welfare; see Spiegler (2016) for a synthesis. Although existing results raise concerns about the performance of markets with price complexity and the impact of competition, they provide relatively limited guidance for competition and consumer protection policy.

This paper aims to inform policy and explores some key questions. Is the degree of complexity a good indicator of market performance? Does an increase in consumer sophistication increase market transparency? Under what conditions does an increase in the number of firms harm consumer welfare? What tests can be used to assess the impact of competition on welfare? Robust answers to these questions are crucial to the practical relevance of extant findings.

The analysis focuses on homogeneous product oligopoly markets where firms compete in price and price complexity. Like in Carlin (2009), complexity makes it harder to assess prices and prevents some consumers from identifying the best deal. Given firms' complexity choices some consumers are 'informed' or 'experts', while others are 'confused'. Like in Varian (1980), the experts are able to assess all prices and buy the lowest-price product, while the confused consumers buy from a randomly selected firm (or make random mistakes). The shares of experts and confused consumers depend on firms' price complexity choices. A unilateral increase in a firm's price complexity increases the share of confused consumers as it raises the difficulty of assessing prices.

Our model generalizes the Varian-Carlin framework to accommodate confusion technologies which may be concave or convex in the aggregate complexity level. In contrast, in Carlin (2009)

\footnotetext{
${ }^{1}$ For instance, Woodward and Hall (2012) show that, in US mortgage markets, deals with the arrangement fee rolled into the interest rate are better than those that quote these fees separately. For a recent discussion of related empirical work, see Campbell (2016).
} 
the confusion technology is linear, while in Varian (1980) the share of confused consumers is exogenous. In the proposed model, the increase in the share of confused consumers triggered by an increase in a firm's complexity may be either reduced or magnified by the complexity of the rivals' price offers. ${ }^{2}$

As a result, this analysis considers a wider gamut of consumer behaviors. For instance, consider a firm which increases its price complexity by including more technical terms or sophisticated jargon. If there is consumer learning by doing, the effect of the increase in complexity would be smaller if rivals' aggregate complexity were higher. Consumers may get better at deciphering technical language the more they are exposed to it, and so there would be some reciprocal cancellation of firms' complexity levels. On the other hand, if consumers are more likely to be demoralized or make mistakes as the informational load increases, the effect of an incremental increase in complexity may be larger when rivals' aggregate complexity is higher.

Consistent with closely related analyses, in our model price complexity underlies consumer heterogeneity and in symmetric equilibrium the firms randomize on prices from a closed interval according to a continuous distribution function. ${ }^{3}$ When setting a relatively low price, a firm benefits from low complexity, but when setting a relatively high price, it benefits from high complexity. As a result, in equilibrium there is a positive relationship between prices and complexity levels, and dispersion in both prices and complexity levels. Despite product homogeneity, prices are strictly above marginal cost and expected profits are strictly positive.

Our extended framework distinguishes between two properties of the confusion technology, the convexity/concavity (the curvature) and the degree of consumer sophistication, and cleanly separates their comparative statics effects. To isolate these properties, our oligopoly model parametrizes the curvature of the confusion technology. However, in the preliminary duopoly analysis, this distinction does not depend on the parametrization of the curvature.

In the symmetric duopoly equilibrium, an increase in the convexity of the confusion technology (or a reduction in its concavity) increases the probability of using low complexity, decreases the lowest price associated with low complexity, weakly increases the lowest price associated with high complexity, decreases the average price and expected industry profit, and increases expected consumer surplus. For a given probability of using low complexity, the expected share of confused consumers decreases in the convexity of the confusion technology. Greater convexity delivers a greater expected market share increment to a firm reducing complexity and competing aggressively. So, as the confusion technology becomes more convex or less concave, the firms rely less on confused consumers and the equilibrium probability of using low complexity increases.

\footnotetext{
${ }^{2}$ One interpretation is that an increase in complexity raises the cost of gathering market-wide information and dissuades more consumers from assessing offers. Then, convexity or concavity in confusion can be related to the convexity or concavity of the cost of gathering market-wide price information. We formalize this interpretation in the online appendix.

${ }^{3}$ These results can be related to the broader literature on price dispersion; see Baye, Morgan, and Scholten (2006) for a survey.
} 
Our duopoly analysis shows that an increase in consumer sophistication decreases the average price and expected firm's and industry's profits, but has no impact on the frequency of using low complexity. This indicates that although consumer protection programmes which raise consumer awareness (e.g., financial literacy programmes) boost consumer surplus, they may not reduce overall market complexity. So, market transparency may not be a good indicator of the effectiveness of consumer awareness initiatives or, more generally, of market performance.

The oligopoly analysis generalizes some of these results and explores the impact of changes in the number of firms and their policy implications. An increase in the number of firms increases a firm's probability of using high complexity and so lowers market transparency. In relatively small markets, expected industry profit is not monotonic in the number of firms. In large markets with concave or linear confusion technology, the expected share of confused consumers and industry profit are bounded away from zero and converge to the highest possible level as the number of firms goes to infinity. In contrast, in large markets with convex confusion technology, the expected number of confused consumers and industry profit converge to zero. Hence, competition policy which increases the number of firms may backfire in relatively small markets and be undesirable in large markets with concave or linear confusion technology.

As concave confusion technologies are consistent with consumer learning by doing, our results suggest that the latter may coexist with poor outcomes for consumers in large markets. However, large markets with convex confusion technologies may perform well. More generally, in markets with many competitors, although firms use high complexity almost surely, the impact of complexity on consumer surplus and an effective policy approach depend crucially on the characteristics of the confusion technology.

Our analysis is closely related to Piccione and Spiegler (2012), Chioveanu and Zhou (2013), and especially, to Carlin (2009). Piccione and Spiegler (2012) explore a general comparability structure in a duopoly market and identify a necessary and sufficient condition for firms to earn max-min profits in equilibrium. In their model, consumers are initially attached to one firm and can only compare offers whose price formats are compatible. In the oligopoly analysis in Chioveanu and Zhou (2013), where firms can choose one of two price formats, an increase in the number of firms induces them to rely more on complexity and may harm consumer welfare. These studies focus on price comparability and how it is affected by price presentation. Closer to Carlin (2009), in our analysis, the share of consumers who gather price information depends on the overall complexity of the market. ${ }^{4}$

Our work complements these analyses by examining the impact of confusion technology's curvature on market outcomes and highlighting its policy implications. The only other analysis of concavity/convexity in confusion technology we are aware of is de Roos (2018), which studies the

\footnotetext{
${ }^{4}$ These papers focus on symmetric market settings. Gu and Wenzel (2014), Chioveanu (2018), and Chioveanu (2019) consider asymmetric duopoly models to study the interplay between price complexity/framing and firm prominence.
} 
impact of limited product comparability on the viability of collusion. His work differs substantially both in focus and in modelling terms: it defines convexity in relation to format similarity rather than overall complexity, it considers repeated interaction, and it explores confusion due to limited comparability.

The next section introduces the model and a taxonomy of confusion technologies. Section 3 presents preliminary results, while section 4 provides a duopoly analysis. Section 5 characterizes the unique symmetric mixed-strategy equilibrium in the general oligopoly model. Section 6 presents comparative statics and convergence results, and discusses consumer protection and competition policy implications. All proofs missing from the text are relegated to the appendix.

\section{Model}

Consider a market for a homogeneous product with $n \geq 2$ identical firms. Marginal costs of production are constant and normalized to zero. There is a unit mass of consumers, each demanding at most one unit of the product and willing to pay at most $v=1 .^{5}$ The firms simultaneously and noncooperatively choose prices, $p_{i}$ for $i \in N=\{1,2, \ldots n\}$, and the complexity of their prices. There are two possible complexity levels and each firm can choose just one of them, $k_{i} \in\{\underline{k}, \bar{k}\}$, where $\bar{k}>\underline{k}$. The profile of firms' price complexity choices is $\mathbf{k}$, an $n$-vector whose $i$-th component is $k_{i}$. Complexity is related to the way in which firms convey price information and can be adjusted as easily as prices, which is reflected by the timing. ${ }^{6}$

In our model, complexity increases the difficulty of assessing firms' price offers and is a source of consumer confusion. Based on firms' complexity choices, there are two types of consumers, experts (or informed) and confused (or uninformed). The experts purchase the cheapest product that provides positive surplus, while the confused consumers buy at random, so that they choose a particular firm with probability $1 / n$.

The firms' price complexity levels determine the share of confused consumers in the market, which is assumed to be a symmetric function. Due to symmetry, for a given $\mathbf{k}$, the shares of experts and confused consumers are solely determined by $n-m$, the count of $\bar{k}$ in $\mathbf{k}$ (or alternatively, by $m$, the count of $\underline{k}$ in $\mathbf{k}$ ) where $m \in\{0,1, \ldots n\}$. Hence, the share of confused consumers can be written as a function,

$$
\gamma: \mathbb{N} \rightarrow[0,1]
$$

Specifically, when $n-m$ firms use $\bar{k}$, the share of confused consumers is $\gamma(n-m)$. For simplicity, let $\gamma(0)=0 .{ }^{7}$ Denote $\gamma(n) \equiv \gamma_{\max }$. The analysis focuses on markets where there are always some

\footnotetext{
${ }^{5}$ The marginal costs and reservation values are normalized for expositional ease and without loss of generality.

${ }^{6}$ Price complexity in this setting is a form of 'price framing'. Alternatively, firms may engage in 'product framing' - a form of spurious product differentiation (e.g., involving decisions on product size or packaging), which cannot be adjusted as frequently as prices and is better captured by a sequential move framework. See the related discussion in Chioveanu and Zhou (2013).

${ }^{7}$ In a market where $\gamma(0) \in(0,1)$, possibly due to the intrinsic characteristics of the product, firms cannot completely eliminate price complexity. So, even when all firms use the simplest price format available, some consumers
} 
expert consumers (i.e., $\gamma_{\max }<1$ ) due to their real-world relevance. However, for completeness it also covers the case where all consumers are confused if all firms use $\bar{k}$ (i.e., $\gamma_{\max }=1$ ).

By making it more difficult to assess firms' offers, an increase in one firm's price complexity increases the fraction of confused consumers in the market, so that

$$
\gamma(n-m)<\gamma(n-m+1)
$$

To capture the impact of a rival's price complexity on the difficulty of evaluating a firm's offer, let

$$
\gamma(n-m)-\gamma(n-m-1)=\frac{\gamma(n-m+1)-\gamma(n-m)}{\beta},
$$

for all $m$ and $\beta>0$. This formulation parametrizes the rate of change of the incremental share of confused consumers, which is summarized by $\beta$, and provides a tractable way to explore more general confusion technologies. If $\beta>1(\beta<1)$, a unilateral incremental increase in complexity is more (less) effective when the rivals' complexity is higher (lower), i.e. firms' complexity levels reinforce (offset) each other in creating confusion. Specifically, depending on the value of $\beta>0$, (2) provides a taxonomy of confusion technologies.

Case 1 Concavity. When $\beta \in(0,1)$, the confusion technology $\gamma$ is concave in the number of complex prices in the market. Formally, (1) and (2) imply that

$$
\gamma(n-m)-\gamma(n-m-1)>\gamma(n-m+1)-\gamma(n-m),
$$

so that a firm's incremental increase in price complexity is more effective in creating confusion when rivals' price complexity is lower (in the sense that fewer competitors employ $\bar{k}$ ).

Case 2 Convexity. When $\beta>1$, the confusion technology $\gamma$ is convex in the number of complex prices in the market. Formally, (1) and (2) imply that

$$
\gamma(n-m)-\gamma(n-m-1)<\gamma(n-m+1)-\gamma(n-m),
$$

so that a firm's incremental increase in price complexity is more effective when rivals' price complexity is higher.

The LHS differences in Cases 1 and 2 capture the increase in the share of confused consumers triggered by a unilateral increase in complexity when $n-m-1$ other firms use $\bar{k}$ (and $m$ rivals use $\underline{k}$ ), while the RHS differences capture the corresponding increase when $n-m$ other firms use $\bar{k}$ (and $m$ rivals use $\underline{k}$ ).

Expression (2) implies that

$$
\gamma(1)=\frac{\gamma(n-m)-\gamma(n-m-1)}{\beta^{n-1-m}}=\frac{\gamma_{\max }-\gamma(n-1)}{\beta^{n-1}} .
$$

get confused. However, this does not change the results qualitatively. 
Then it follows that

$$
\gamma(n-m)=\frac{\gamma_{\max }\left(\beta^{n-m}-1\right)}{\beta^{n}-1} \text { for } \beta \neq 1
$$

The share of confused consumers for given $n-m$ can be expressed in terms of the primitives of the model: the market structure $(n)$, the curvature of the confusion technology $(\beta)$, and the highest possible share of confused consumers $\left(\gamma_{\max }\right)$. In our model, ceteris paribus, changes in $\gamma_{\max }$ trigger changes in $\gamma(n-m)$ for any $n-m$. For this reason, $\gamma_{\max }$ can be used as a measure of the degree of consumer sophistication, with a higher value corresponding to lower sophistication. ${ }^{8}$

Case 3 Linearity. When $\beta=1$, the price complexity of one firm does not affect the effectiveness of an increase in a rival's complexity. Formally, (1) and (2) imply that

$$
\gamma(n-m)-\gamma(n-m-1)=\gamma(n-m+1)-\gamma(n-m) .
$$

In this case, which is closely related to Carlin (2009), (3) is replaced by

$$
\gamma(n-m)=\frac{\gamma_{\max }(n-m)}{n} \text { for } \beta=1 .
$$

The main analysis focuses on $\beta \neq 1$, but a characterization of the equilibrium for $\beta=1$ is included at the end of section 5 and the policy discussion in section 6 covers all cases.

As the share of confused consumers is assumed to be a symmetric function of firms' price complexity levels, in the setting above it is expressed as a function of the count of $\bar{k}$ in $\mathbf{k}$. Alternatively, the share of confused consumers could be written as a function of the profile of firms' price complexity choices, $\varphi(\mathbf{k})$. Then, Case 1 corresponds to a situation where there is substitutability in confusion (i.e., the confusion technology $\varphi$ satisfies strictly decreasing differences), while Case 2 corresponds to a situation where there is complementarity in confusion (i.e., the confusion technology $\varphi$ satisfies strictly increasing differences). ${ }^{9}$ Further details are relegated to the online appendix.

Our reduced form model accommodates various interpretations. It can be related to an environment where consumers have limited time and incur a time cost to assess firms' offers, so that they use a two-step approach. Depending on the available time and the overall market complexity, they first decide whether to collect information on prices or not. They can directly observe the overall market complexity, e.g., they may receive information amalgamated by the media or social networks or transmitted via word-of-mouth. However, they do not observe individual firms' complexity levels unless they decide to collect information. Complexity focuses consumers' attention to this particular aspect and may dissuade them from gathering price information. The consumers who decide to gather information buy the lowest-price product and the others select a product

\footnotetext{
${ }^{8}$ It can also be shown that if $\gamma(0) \in(0,1)$, the share of confused consumers when exactly $n-m$ firms use $\bar{k}$ is equal to the expression in (3) plus a constant equal to $\gamma(0)$.

${ }^{9}$ See Vives (1999, Ch. 2) for general discussion of strategic substitutability and complementarity which relates to profits. However, here, substitutability and complementarity do not relate to firms' profits.
} 
randomly. The online appendix formalizes the interpretation of consumer confusion rooted in the cost of processing information and provides further discussion.

Our analysis is related to Piccione and Spiegler (2012) and Chioveanu and Zhou (2013). However, these papers analyze the impact of price complexity (and, more generally, framing) on the price comparability structure. In contrast, the interpretation presented above focuses on markets where consumers use a two-stage decision making process. Here, by making gains from comparisons or switching less obvious, complexity may discourage information gathering or reinforce inertia. Our model is also consistent with a default-bias interpretation, whereby each consumer is initially assigned to one firm and the firms have equal bases of consumers ex-ante. In this case, confused consumers uphold their default option, while the experts select the cheapest alternative.

\section{Preliminary Findings}

Given competitors' price and price complexity choices $\left(\mathbf{p}_{-i}, \mathbf{k}_{-i}\right)$, firm $i$ 's ex post profit can be written as

$$
\pi_{i}\left(p_{i}, k_{i}, \mathbf{p}_{-i}, \mathbf{k}_{-i}\right)=p_{i} \cdot\left[\frac{\gamma(n-m)}{n}+q_{i}\left(p_{i}, \mathbf{p}_{-i}\right)(1-\gamma(n-m))\right],
$$

where firm $i$ 's share of informed consumers, $q_{i}\left(p_{i}, \mathbf{p}_{-i}\right)$, is given below.

$$
q_{i}\left(p_{i}, \mathbf{p}_{-i}\right)= \begin{cases}1 & \text { if } p_{i}<\min \left\{p_{j}, 1\right\} \text { for } j \in N, j \neq i \\ \frac{1}{|H|} & \text { if } p_{i}=p_{j} \leq 1, \text { for } j \in H \subseteq N, \text { and } p_{l}>p_{i}, \text { for } l \in N \backslash H, j, l \neq i \\ 0 & \text { if } p_{i}>\min \left\{p_{j}, 1\right\} \text { for } j \in N, j \neq i .\end{cases}
$$

The first term in square brackets in $\pi_{i}$ is firm $i$ 's share of confused consumers, while the second term is firm $i$ 's share of experts. All experts buy from firm $i$ if its price is strictly lower than rivals' prices. If firm $i$ and other firms tie at the lowest price, then they share equally the experts. If at least one firm $j \neq i$ offers a price $p_{j}$ lower than $p_{i}$, then firm $i$ does not sell to experts.

Although confused consumers cannot assess prices, they do not pay more than their valuation. A justification for this assumption is that, if firms charge prices above $v=1$, the consumers would realize at checkout (or after purchase) and decline the purchase (or return the product free of cost). Therefore, the firms have no incentives to charge prices in excess of consumers' willingness to pay. ${ }^{10}$

We first explore pure complexity strategy equilibria. If all firms choose $\underline{k}$ for sure, they make zero profits. But then a unilateral deviation to $\bar{k}$ allows a firm to make positive profit on its confused consumer base. If some firms use $\bar{k}$ and others use $\underline{k}$, there are both expert and confused consumers. Varian (1980) implies that the firms mix on prices from a closed interval. But if a $\bar{k}$-firm deviates to $\underline{k}$ and sets the lowest price, it makes higher profit. At that price, the firm serves

\footnotetext{
${ }^{10}$ However, it can be shown that our results are robust in a setting where confused consumers may be able to pay $v>1$, for $v$ small enough.
} 
all the experts and, although the deviation to $\underline{k}$ decreases the firm's confused consumers base, this loss is more than compensated by the gain in the share of experts. The proof of the following lemma formalizes and completes these arguments.

Lemma 1 There is no equilibrium where all firms use pure price complexity strategies.

Proof. (a) Suppose that $k_{i}=\underline{k}$ for all $i \in N$ (i.e., $\gamma(0)=0$ ). Then, firms compete à la Bertrand and make zero profit. If a firm unilaterally deviates to $\bar{k}$ and price $p \in(0,1)$, it makes strictly positive profit. A contradiction.

(b) Suppose exactly $n-m$ firms use $\bar{k}$ (for $m \geq 1$ ). (a) implies that $m<n$. Then there are $1-\gamma(n-m)$ experts and $\gamma(n-m)>0$ confused consumers, and the results in Varian (1980) apply. The unique candidate equilibrium dictates mixed strategy pricing according to a c.d.f. on $\left[p_{0}, 1\right]$ and each firm's expected profit is $\gamma(n-m) / n=p_{0}[1-\gamma(n-m)(n-1) / n]$. But, if one of the $n-m$ firms choosing $\bar{k}$ deviates to $\left(p_{0}, \underline{k}\right)$ it makes profit $p_{0}[1-\gamma(n-m-1)(n-1) / n]>$ $p_{0}[1-\gamma(n-m)(n-1) / n]$.

(c) Suppose all firms choose $\bar{k}$ for sure (that is, there are $\gamma(n)=\gamma_{\max }$ confused consumers). If $\gamma_{\max }<1$, the argument in (b) applies unchanged. If $\gamma_{\max }=1$, then in the unique candidate equilibrium $p_{i}=1$ and $\pi_{i}=1 / n$ for all $i$. But then if a firm unilaterally deviates to $\underline{k}$ and price $p_{i}=1-\varepsilon$, it obtains profit $(1-\varepsilon)[\gamma(n-1) / n+(1-\gamma(n-1))]>1 / n$ for $\varepsilon<(1-\gamma(n-1)) /$ $[1 /(n-1)+(1-\gamma(n-1))]$.

Lemma 1 implies that in any candidate equilibrium at least one firm randomizes its price complexity. Therefore, with positive probability the firms face both experts and confused consumers. The conflict between the incentives to fully exploit confused consumers and to vigorously compete for the experts rules out equilibria involving pure price strategies; see Varian (1980) and Rosenthal (1980). The proof of the following result is standard and therefore omitted.

Lemma 2 There is no equilibrium where all firms use pure pricing strategies.

Lemmas 1 and 2 show that in any equilibrium there must be dispersion in both price complexities and prices. A firm's strategy space is $[0,1] \times\{\underline{k}, \bar{k}\}$. Denote by $\xi_{i} \equiv \xi_{i}\left(p_{i}, k_{i}\right)$ firm $i$ 's mixed strategy over price and price complexity. This analysis focuses on symmetric mixed strategies, where $F(p)$ is the marginal c.d.f. of firms' random prices, defined on an interval $S \subseteq[0,1]$, and $\lambda(p)$ and $1-\lambda(p)$ are the probabilities that price $p \in S$ is associated with complexity level $\underline{k}$ and $\bar{k}$, respectively. Then, the overall probability of using $\underline{k}$ is $\int_{p \in S} \lambda(p) d F(p) \in(0,1)$.

The following result presents properties of the firms' pricing strategies.

Lemma 3 In symmetric equilibrium, (i) the support of the pricing distribution $(S)$ is a connected interval; (ii) if $\gamma_{\max }<1$ the pricing c.d.f. is continuous everywhere, while if $\gamma_{\max }=1$, it is continuous everywhere except possibly at the upper bound of $S$; and (iii) inf $S=p_{0}>0$ and $\sup S=1$. 
The next sections build on these results to characterize the symmetric Nash equilibrium in mixed strategies. Section 4 illustrates the approach in a simple duopoly model and presents some preliminary comparative statics results. Section 5 analyzes a general oligopoly model, while section 6 discusses competition and consumer protection policy implications, by combining analytical results and numerical simulations.

\section{Duopoly Analysis}

Consider a duopoly market and suppose firm $j \neq i$ follows the mixed strategy $\xi_{j}$ presented in section 3. At a given price $p_{j}$, firm $j$ uses low complexity $\underline{k}$ with probability $\lambda\left(p_{j}\right)$ and high complexity with probability $1-\lambda\left(p_{j}\right)$. Firm $j$ 's price is a random draw from its price c.d.f. Then, firm $i$ 's profit at price $p \geq p_{0}=\min S>0$ and complexity $\underline{k}$ is given by

$$
\begin{aligned}
\pi_{i}(p, \underline{k})= & p \int_{p}^{1}\left[\lambda\left(p_{j}\right)(1-\gamma(0))+\left(1-\lambda\left(p_{j}\right)\right)(1-\gamma(1))\right] d F\left(p_{j}\right)+ \\
& \frac{p}{2} \int_{p_{0}}^{1}\left[\lambda\left(p_{j}\right) \gamma(0)+\left(1-\lambda\left(p_{j}\right)\right) \gamma(1)\right] d F\left(p_{j}\right) .
\end{aligned}
$$

For a given realization of rival's price $p_{j}$, the first square bracket gives the expected share of experts. If firm $j$ also uses $\underline{k}$, which happens with probability $\lambda\left(p_{j}\right)$, the expert share is $(1-\gamma(0))$; if, instead, it uses $\bar{k}$, which happens with probability $1-\lambda\left(p_{j}\right)$, the expert share is $(1-\gamma(1))$. Similarly, for a given $p_{j}$, the second square bracket gives the expected share of confused consumers. As firm $j$ 's price $p_{j}$ is a random draw, these expected shares are integrated over the relevant realizations of $p_{j}$. Firm $i$ serves experts whenever the rival's price is higher $\left(p_{j}>p\right)$, whereas it serves half of the confused consumers for all $p_{j}$ 's.

Firm $i$ 's profit at price $p$ and complexity $\bar{k}$ is

$$
\begin{aligned}
\pi_{i}(p, \bar{k})= & p \int_{p}^{1}\left[\lambda\left(p_{j}\right)(1-\gamma(1))+\left(1-\lambda\left(p_{j}\right)\right)(1-\gamma(2))\right] d F\left(p_{j}\right)+ \\
& \frac{p}{2} \int_{p_{0}}^{1}\left[\lambda\left(p_{j}\right) \gamma(1)+\left(1-\lambda\left(p_{j}\right)\right) \gamma(2)\right] d F\left(p_{j}\right) .
\end{aligned}
$$

The terms in square brackets present the expected shares of experts and, respectively, confused consumers for a given realization of rival's price $p_{j}$. In this case, if firm $j$ chooses the low complexity $\underline{k}$ - which happens with probability $\lambda\left(p_{j}\right)$ - there are $(1-\gamma(1))$ experts and $\gamma(1)$ confused consumers. If firm $j$ chooses the high complexity $\bar{k}$ - which happens with probability $1-\lambda\left(p_{j}\right)$ - then there are $(1-\gamma(2))=\left(1-\gamma_{\max }\right)$ experts and $\gamma(2)=\gamma_{\max }$ confused consumers. Firm $j$ 's price $p_{j}$ is a random draw from its pricing c.d.f. The share of experts is integrated over all the price realizations where firm $i$ serves these consumers (i.e., for all $p_{j}>p$ ). Firm $i$ serves half of the confused consumers for all $p_{j}$ 's.

Substituting $\gamma(0)=0$ and using (3), (5) and (6), the incremental profitability of an increase in complexity is

$$
\pi_{i}(p, \bar{k})-\pi_{i}(p, \underline{k})=\frac{p \gamma(1)}{2}\left(-\int_{p}^{1} \Lambda\left(p_{j}\right) d F\left(p_{j}\right)+\int_{p_{0}}^{p} \Lambda\left(p_{j}\right) d F\left(p_{j}\right)\right),
$$


where $\Lambda\left(p_{j}\right) \equiv\left[1+\left(1-\lambda\left(p_{j}\right)\right)(\beta-1)\right]>0$. Evaluating (7) at $p=p_{0}$ and $p=1$ gives, respectively,

$$
\frac{p_{0} \gamma(1)}{2}\left(-\int_{p_{0}}^{1} \Lambda\left(p_{j}\right) d F\left(p_{j}\right)\right)<0 \text { and } \frac{\gamma(1)}{2}\left(\int_{p_{0}}^{1} \Lambda\left(p_{j}\right) d F\left(p_{j}\right)\right)>0 .
$$

Expression (7) is strictly increasing in $p$ : the first term in brackets (which is negative) decreases as $p$ increases (the integration range gets smaller), whereas the second term in brackets (which is positive) increases as $p$ increases (the integration range gets wider).

To maximize its expected profit, firm $i$ chooses

$$
k(p)=\left\{\begin{aligned}
& \underline{k} \text { if } p<\hat{p}, \\
& \bar{k} \text { if } p>\hat{p}, \\
& k, \forall k \in\{\underline{k}, \bar{k}\} \text { if } p=\hat{p},
\end{aligned}\right.
$$

where the threshold price $\hat{p}$ follows from equating (7) to zero. So, in symmetric equilibrium, prices below a cut-off level $\hat{p}>p_{0}$ are associated with $\underline{k}$, while price above $\hat{p}$ are associated with $\bar{k}$, and each firm assigns probability $\lambda=\int_{p_{0}}^{1} \lambda(p) d F(p)=F(\hat{p}) \in(0,1)$ to low complexity $(\underline{k})$ and $1-\lambda$ to high complexity $(\bar{k})$. A formal proof of this result is presented in the next section for the general oligopoly model.

Suppose now that firm $j \neq i$ uses the price complexity strategy identified above and that $F(p)$ satisfies Lemma 3. Then, firm $i$ 's expected profits at $p=p_{0}$ and when $p \rightarrow \hat{p}$ are

$$
\pi_{i}\left(p_{0}, \underline{k}\right)=p_{0}\left[\lambda+\frac{(1-\lambda)(2-\gamma(1))}{2}\right] \text { and } \lim _{p \rightarrow \hat{p}} \pi_{i}(p, \underline{k})=\frac{\hat{p}}{2}(1-\lambda)(2-\gamma(1)),
$$

whereas firm $i$ 's expected profits at $p=\hat{p}$ and $p=1$ are

$$
\pi_{i}(\hat{p}, \bar{k})=\frac{\hat{p}}{2}\left[2(1-\lambda)+\lambda \gamma(1)-(1-\lambda) \gamma_{\max }\right] \text { and } \pi_{i}(1, \bar{k})=\frac{1}{2}\left[\lambda \gamma(1)+(1-\lambda) \gamma_{\max }\right]
$$

The expected profit for an arbitrary price $p$ is presented in the appendix. The constant profit conditions imply that in duopoly equilibrium the probability of using $\bar{k}$ and a firm's expected profit are given by

$$
1-\lambda=\frac{1}{\beta+1} \in(0,1) \text { and } \pi=\frac{\gamma_{\max }(2 \beta+1)}{2(\beta+1)^{2}},
$$

while the boundary prices $p_{0}$ and $\hat{p}$ are

$$
p_{0}=\frac{\gamma_{\max }(2 \beta+1)}{2(\beta+1)^{2}-\gamma_{\max }} \in(0,1) \text { and } \hat{p}=\frac{\gamma_{\max }(2 \beta+1)}{2(\beta+1)-\gamma_{\max }} \in(0,1]
$$

Note that $\hat{p}=1$ iff $\gamma_{\max }=1$.

Finally, $F(p)$ is implicitly determined by $\pi(\underline{k}, p)=\pi$ and $\pi(\bar{k}, p)=\pi$, and presented in the appendix. For $\gamma_{\max }=1$, the price distribution has a mass point at the upper bound of its support as $(1-F(1))=1 /(\beta+1)$.

Proposition 1 In the unique symmetric duopoly equilibrium, firms choose prices randomly according to the c.d.f. $F(p)$ presented in (A1). Prices on $\left[p_{0}, \hat{p}\right)$ are associated with low price complexity $(\underline{k})$, while prices on $[\hat{p}, 1]$ are associated with high price complexity $(\bar{k})$, with $p_{0}$ and $\hat{p}$ defined in (10). Each firm chooses $\underline{k}$ with probability $\lambda=F(\hat{p})$ and $\bar{k}$ with probability $1-\lambda$, where $\lambda$ is presented in (9), along with a firm's expected equilibrium profit, $\pi$. 
For $\gamma_{\max }<1$, symmetric equilibrium price c.d.f. is continuous everywhere. In contrast, for $\gamma_{\max }=1, \hat{p}=1$ and the equilibrium price distribution is continuous for all $p<1$, but has an atom at the upper bound as $\lim _{p \nearrow 1} F(p)=\beta /(\beta+1)<F(1)=1 .{ }^{11}$ There is dispersion in both prices and complexity levels, a positive relationship between prices and complexity, and the firms' strategies and market outcomes depend on both $\beta$ and $\gamma_{\max }$.

Figure 1 illustrates symmetric equilibrium price c.d.f.s for given $\gamma_{\max }$ and two values of $\beta$. Solid lines correspond to prices associated with $\underline{k}$, while dashed lines correspond to $\bar{k}$.

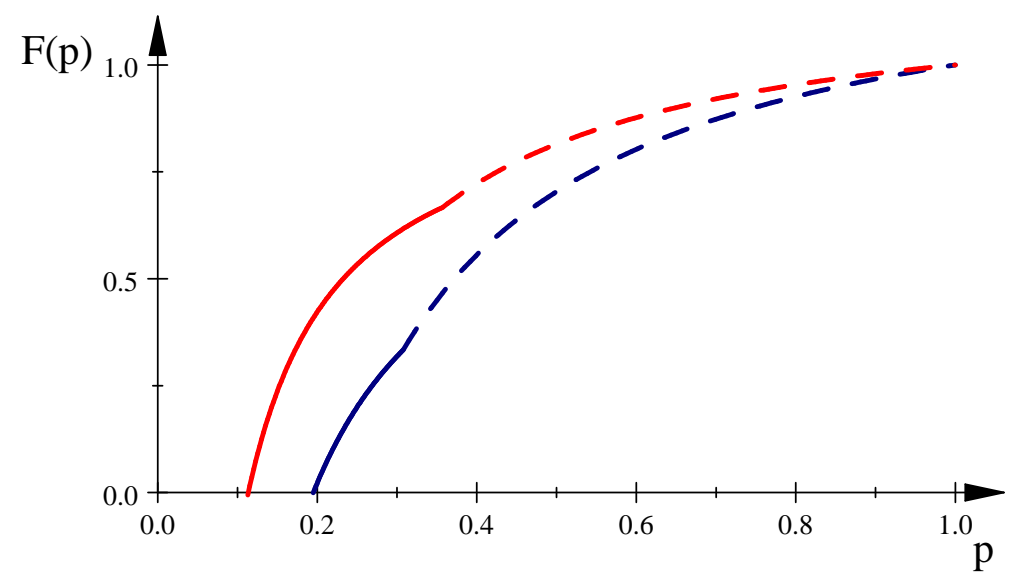

Figure 1: Symmetric equilibrium pricing distributions for $\gamma_{\max }=.4$. The lower c.d.f. is for $\beta=1 / 2$ (with $p_{0}=0.195, \hat{p}=0.307$, and $\pi=0.178$ ). The upper c.d.f. is for $\beta=2$ (with $p_{0}=0.113, \hat{p}=0.357$ and $\left.\pi=0.111\right)$.

Our analysis distinguishes between two properties of the confusion technology, the convexity/concavity (captured by $\beta$ ) and consumer sophistication (captured by $\gamma_{\max }$ ), and separates their comparative statics effects. In duopoly, the curvature is given by a unique ratio, $\beta=\left(\gamma_{\max }-\gamma(1)\right) /$ $(\gamma(1)-\gamma(0))$ - see (2) for $n=2$ - and the separation is straightforward. Although this is no longer true with more than two firms, as we parametrize the curvature of the confusion technology, the effects of $\beta$ and $\gamma_{\max }$ can still be isolated in our oligopoly model.

In duopoly, for $\beta \in(0,1)$, an increase in $\beta$ translates into a reduction in the concavity of the confusion technology: a firm's attempt to confuse consumers is thwarted to a lesser extent by rival's use of high complexity. In contrast, for $\beta>1$, an increase in $\beta$ corresponds to an increase in the convexity of the confusion technology, and so a firm's attempt to confuse is magnified to a higher extent by the rival's use of high complexity.

Holding the curvature $(\beta)$ fixed, expression (3) shows that with duopoly a change in the share of confused consumers when both firms use $\bar{k}$ (i.e., a change in $\gamma_{\max }$ ) triggers a change in the share of confused consumers when only one firm uses $\bar{k}(\gamma(1)) .{ }^{12}$

\footnotetext{
${ }^{11}$ When $\gamma_{\max }=1, \hat{p}=1$ so $\lim _{p \nearrow 1} \pi_{i}(p, \underline{k})=\pi_{i}(1, \bar{k})$ and all the mass placed on $\bar{k}$ is clustered at the upper bound of the support.

${ }^{12}$ As in duopoly $\beta=\left(\gamma_{\max }-\gamma(1)\right) /(\gamma(1)-\gamma(0))$, then $\gamma(1)=\gamma_{\max } /(\beta+1)$.
} 
Corollary 1 In the symmetric duopoly equilibrium, the probability of using the low price complexity $(\lambda)$ increases in $\beta$, the lower bound of the pricing c.d.f. $\left(p_{0}\right)$ decreases in $\beta$, the cut-off price $(\hat{p})$ increases in $\beta$ for $\gamma_{\max }<1$ and is independent of $\beta$ for $\gamma_{\max }=1$, while the expected individual profit $(\pi)$, the expected industry profit $(2 \pi)$, the expected share of confused consumers, and the average price decrease in $\beta$.

An increase in the curvature of the confusion technology $(\beta)$ leads to more market transparency and stronger competition: the firms put higher probability on $\underline{k}$ and the average price and expected industry profit decrease. However, an increase in $\beta$ also increases price dispersion (as $p_{0}$ decreases). As total welfare is constant (and normalized to one), the decrease in expected industry profit corresponds to an equal increase in expected consumer surplus. Figure 2 illustrates how the probability of using $\underline{k}$ and expected industry profit vary with $\beta$, for given $\gamma_{\max }$.

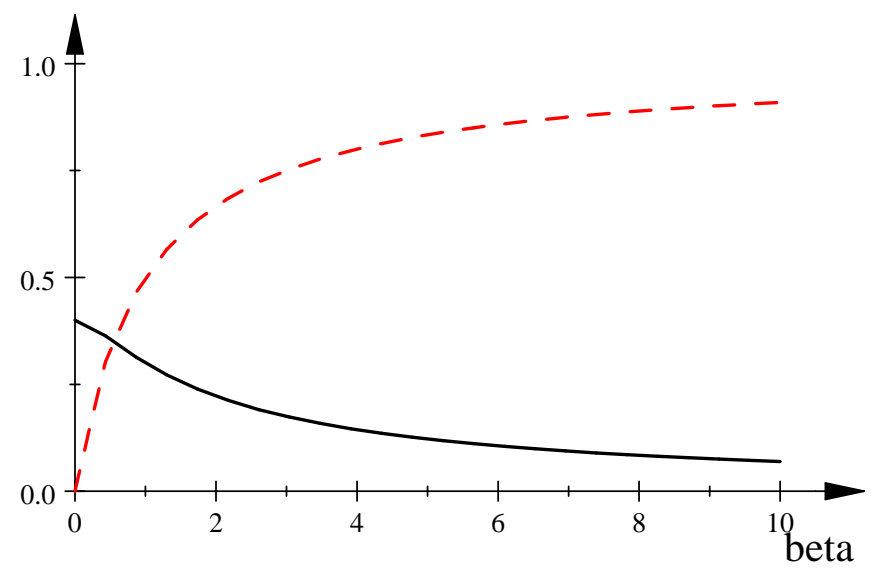

Figure 2: Probability of using $\underline{k}$ (dash, increasing) and expected industry profit (solid, decreasing) as functions of $\beta$ for $n=2$ and $\gamma_{\max }=0.4$.

For intuition on why the curvature makes a difference, take a situation where both firms use $\bar{k}$ and so the share of confused consumers is $\gamma_{\max }$. As both firms use $\bar{k}$, they set prices above $\hat{p}$. Consider a unilateral deviation from $\bar{k}$ to $\underline{k}$ and a price below $\hat{p}$, where the deviator aims to attract the experts. In duopoly $\beta=\left(\gamma_{\max }-\gamma(1)\right) /(\gamma(1)-\gamma(0))$ and, as $\gamma(0)=0$, this deviation reduces the share of confused consumers to $\gamma(1)=\gamma_{\max } /(\beta+1)$. Clearly, as $\beta$ increases, the deviation is more effective as the reduction in the confused consumer share is larger.

Take now a situation where both firms use $\underline{k}$ and so the share of confused consumers is $\gamma(0)=0$. As both firms use $\underline{k}$, they set prices below $\hat{p}$. Consider a unilateral deviation from $\underline{k}$ to $\bar{k}$ and a price above $\hat{p}$. The deviator targets the confused consumers and the deviation increases the share of confused consumers to $\gamma(1)=\gamma_{\max } /(\beta+1)>0$. As $\beta$ increases, the deviation is less effective as the increase in the confused consumer share is smaller.

Consider a situation where one firm uses $\underline{k}$ and the other $\bar{k}$. The $\bar{k}$ - firm sells only to its share of confused consumers, that is, to $\gamma(1) / 2=\gamma_{\max } / 2(\beta+1)$ consumers (this is because its price is higher than $\hat{p}$, while the rival's price is lower than $\hat{p}$ ). If this firm deviates to $\underline{k}$ and a lower price, its expected market share is $1 / 2$. So, the market share gain from the deviation is $\beta / 2(\beta+1)$ and is 
increasing in the convexity of the confusion technology, $\beta$. Combining the three arguments, firms always have a greater incentive to choose low complexity when $\beta$ is higher.

For a more formal analysis of the role of $\beta$, consider firm $i$ 's incentives at the cut-off price $\hat{p}$. At this price, firm $i$ is indifferent between using low and high price complexity. Proposition 1 implies that when firm $i$ sets price $\hat{p}$, firm $j$ has a higher price for sure when it uses $k_{j}=\bar{k}$, whereas firm $j$ has a lower price for sure when it chooses $k_{j}=\underline{k}$. So, when firm $i$ sets price $\hat{p}$, the experts buy from firm $i$ if firm $j$ chooses $\bar{k}$ and they buy from firm $j$ if firm $j$ chooses $\underline{k}$. Then, if the rival uses $\bar{k}$ - which happens with probability $(1-\lambda)$ - when firm $i$ increases its complexity from $\underline{k}$ to $\bar{k}$, there is a reduction in the share of experts and an increase in firm $i$ 's share of confused consumers. The net (negative) change in firm $i$ 's market share is

$$
\left[\left(1-\gamma_{\max }\right)-(1-\gamma(1))\right]+\frac{\gamma_{\max }-\gamma(1)}{2}=-\gamma_{\max }\left[\frac{\beta}{2(\beta+1)}\right] \equiv \Gamma_{1},
$$

where the equality follows from (3). If the rival uses $\underline{k}$ - which happens with probability $(\lambda)$ - when firm $i$ increases its complexity from $\underline{k}$ to $\bar{k}$, the positive change in firm $i$ 's market share is

$$
\frac{\gamma(1)-\gamma(0)}{2}=\gamma_{\max }\left[\frac{1}{2(\beta+1)}\right] \equiv \Gamma_{2}
$$

In equilibrium, firm $j$ 's probability of choosing $\underline{k}(\lambda)$ balances the expected loss and the expected gain, that is, $(1-\lambda) \Gamma_{1}+\lambda \Gamma_{2}=0$. Keeping $\lambda$ fixed, following an increase in $\beta$, as both $d \Gamma_{1} / d \beta<0$ and $d \Gamma_{2} / d \beta<0$, firm $i$ strictly prefers $\underline{k}$ to $\bar{k}$ at price $\hat{p}$ and so the price where firm $i$ is indifferent between $\underline{k}$ and $\bar{k}$ is strictly higher than $\hat{p}$. Therefore, following an increase in $\beta$, a firm has a stronger incentive to use $\underline{k}$, which underlies the comparative statics of $\lambda$ with respect to $\beta$ in Corollary 1. In duopoly markets, the generality of this argument can be highlighted by using the LHS terms in (11) and (12) to write $(1-\lambda) \Gamma_{1}+\lambda \Gamma_{2}=0$ as

$$
-(1-\lambda)\left(\frac{\gamma_{\max }-\gamma(1)}{\gamma(1)-\gamma(0)}\right)+\lambda=0 \Leftrightarrow-(1-\lambda) \beta+\lambda=0
$$

where the fraction measures the concavity/convexity of the confusion technology (and is equal to $\beta$ ). Using the implicit function theorem, this expression shows that an increase in the convexity (or decrease in the concavity) of the confusion technology will trigger an increase in $\lambda$.

Note also that when fixing $\lambda$, an increase in convexity (decrease in concavity) leads to a decrease in the expected share of confused consumers. ${ }^{13}$ This decrease makes the firms rely less on confused consumers and increases their incentives to use low complexity, triggering an increase in the equilibrium value of $\lambda$.

While average prices decrease in $\beta$, it is less obvious how the average price conditional on low complexity varies with $\beta$. For instance, in Figure 1, an increase in $\beta$ from $1 / 2$ to 2 triggers an increase in the cumulative probability at each price $p$ which was assigned positive density for $\beta=1 / 2$ and also a decrease in $p_{0}$. These two effects indicate that the average price for $\beta=2$

\footnotetext{
${ }^{13}$ The expected share of confused consumers for given $\lambda$ is $\sum_{m=0}^{2} C_{2}^{m} \lambda^{m}(1-\lambda)^{2-m} \gamma(2-m)=\gamma_{\max }\left[\beta(1-\lambda)^{2}+\right.$ $\left.1-\lambda^{2}\right] /(\beta+1)$ and it is easy to check that it decreases in $\beta$ for $\lambda \in(0,1)$.
} 
is lower than that for $\beta=1 / 2$. However, the change in the average price conditional on low complexity is affected by an additional effect which partly offsets these two, as the cut-off price $\hat{p}$ increases in $\beta$. Using (A1), the price distribution conditional on the price being below the cut-off value $\hat{p}$ and the corresponding conditional expected price are presented in the appendix. It can be checked that the expected price conditional on low complexity is not generally monotonic in $\beta$. For instance, for $\gamma_{\max }=0.4$, it increases for low values of $\beta$ and then decreases, while for $\gamma_{\max }=$ 1 , it monotonically decreases in $\beta .^{14}$

Corollary 2 In symmetric duopoly equilibrium, the probability of using low price complexity ( $\lambda$ ) is independent of $\gamma_{\max }$, while the lower bound of the pricing c.d.f. $\left(p_{0}\right)$, the cut-off price $(\hat{p})$, expected individual profit $(\pi)$, expected industry profit $(2 \pi)$, expected share of confused consumers, the average price, and the average price conditional on low complexity, all increase in $\gamma_{\max }$.

As discussed below Corollary 1, the equilibrium $\lambda$ makes a firm indifferent between low and high price complexity at $\hat{p}$. To isolate the comparative statics effect of $\gamma_{\max }$, the concavity/convexity of the confusion technology $\left(\beta=\left(\gamma_{\max }-\gamma(1)\right) /(\gamma(1)-\gamma(0))\right.$ is held constant. Then, as it can be seen from expressions (11) and (12), consumer sophistication $\left(\gamma_{\max }\right)$ can be factored out of $(1-\lambda) \Gamma_{1}+\lambda \Gamma_{2}=0$, and so the equilibrium value of $\lambda$ is independent of $\gamma_{\max }$.

An implication of Corollary 2, is that consumer policies which increase the degree of consumer sophistication, for instance, financial literacy programmes, decrease the average prices and the expected share of confused consumers, and increase expected consumer surplus. However, they increase price dispersion (by decreasing the lower bound of the pricing support, $p_{0}$ ) and we identify the possibility that they may not affect market transparency as reflected by firms' probability of choosing low complexity. These results suggest that market transparency and price dispersion may not be good indicators of the success of consumer education programmes or of market performance.

\section{Oligopoly Equilibrium}

This section characterizes the symmetric equilibrium in oligopoly markets. It mainly focuses on a framework where the confusion technology is either concave or convex in firms' price complexity levels (where $\beta \lessgtr 1$ ), but also includes a variant without second order effects $(\beta=1$ ).

Consider an arbitrary firm $i \in N$. Suppose that firm $i$ 's competitors follow the mixed strategy presented in section 3 . Then, the competitors choose prices randomly from a distribution function $F(p)$ which satisfies Lemma 3, the probability that a (random) price $p$ is associated with the low complexity $\underline{k}$ is given by $\lambda(p)$, and the probability that $p$ is associated with high complexity $\bar{k}$ is $1-\lambda(p)$. Although firm $i$ 's competitors choose prices from the same c.d.f., their price draws may be different and so the probabilities of using $\underline{k}$ may also be different across these firms.

Let $\wp\left(N_{-i}\right)$ be the power set of $N_{-i}=N \backslash\{i\}$ and $M_{m}=\left\{M \subset \wp\left(N_{-i}\right)|| M \mid=m\right\} \cdot \wp\left(N_{-i}\right)$ is the set of all subsets of $N_{-i}$ and $M_{m}$ is the set of all subsets of $\wp\left(N_{-i}\right)$ of cardinality $m$. Consider

\footnotetext{
${ }^{14}$ When $\gamma_{\max }=0.4$, the expected price conditional on low complexity is 0.25115 for $\beta=0.05,0.25149$ for $\beta=0.15$, and 0.24292 for $\beta=0.5$.
} 
competitors' ex post price profile $\mathbf{p}_{-i} \in S^{n-1}$ and let $p_{j}$ and $p_{l}(j, l \neq i)$ stand for elements of $\mathbf{p}_{-i}$. Denote by

$$
\mathrm{P}_{n-1}^{m}=\sum_{M \subset M_{m}}\left[\prod_{j \in M} \lambda\left(p_{j}\right) \prod_{k \in N_{-i} \backslash M}\left(1-\lambda\left(p_{l}\right)\right)\right],
$$

the overall probability that out of $n-1$ rivals exactly $m \geq 0$ use $\underline{k}$ and $n-m-1$ use $\bar{k}$.

Example 1 Let $n=3, N_{-i}=\{j, l\}$, and $\mathbf{p}_{-i}=\left(p_{j}, p_{l}\right)$. Using (13), $\mathrm{P}_{2}^{2}=\lambda\left(p_{j}\right) \lambda\left(p_{l}\right), \mathrm{P}_{2}^{1}=$ $\left[\lambda\left(p_{j}\right)\left(1-\lambda\left(p_{l}\right)\right)+\left(1-\lambda\left(p_{j}\right)\right) \lambda\left(p_{l}\right)\right]$, and $\mathrm{P}_{2}^{0}=\left(1-\lambda\left(p_{j}\right)\right)\left(1-\lambda\left(p_{l}\right)\right)$. In this case, $\wp\left(N_{-i}\right)=$ $\{\varnothing,\{j\},\{l\},\{j, l\}\}, M_{2}=\{j, l\}, M_{1}=\{\{j\},\{l\}\}$, and $M_{0}=\{\varnothing\}$.

Using (13), firm $i$ 's expected profit at price $p \geq p_{0}=\min S>0$ and complexity $\underline{k}$ is given by

$$
\begin{aligned}
\pi_{i}(p, \underline{k})= & p \int_{p}^{1} \ldots \int_{p}^{1}\left[\sum_{m=0}^{n-1} \mathrm{P}_{n-1}^{m}(1-\gamma(n-m-1))\right] d F\left(p_{j}\right) \ldots d F\left(p_{l}\right)+ \\
& \frac{p}{n} \int_{p_{0}}^{1} \ldots \int_{p_{0}}^{1}\left[\sum_{m=0}^{n-1} \mathrm{P}_{n-1}^{m} \gamma(n-m-1)\right] d F\left(p_{j}\right) \ldots d F\left(p_{l}\right) .
\end{aligned}
$$

The first term in $\pi_{i}(p, \underline{k})$ corresponds to firm $i$ 's profit on the expert consumer segment. When out of $n-1$ rivals exactly $m \geq 0$ use $\underline{k}$ and $n-m-1$ use $\bar{k}$, given that firm $i$ also employs $\underline{k}$, there are $\gamma(n-m-1)$ confused consumers and $(1-\gamma(n-m-1))$ experts. The first term in square brackets sums up over all values of $m$. Firm $i$ serves experts only if its price is lower than rivals' prices, which is reflected when integrating over $p_{j}>p$ for all $j \neq i$. The second term is firm $i$ 's profit on the confused consumer segment. Firm $i$ serves a share $1 / n$ of these consumers. The second term in square brackets sums the corresponding share of confused consumers $\gamma(n-m-1)$ over all values of $m$. Firm $i$ serves its share of confused consumers regardless of rivals' prices, which is reflected by integrating over $p_{j}>p_{0}$ for all $j \neq i$.

Using (13), firm $i$ 's expected profit at price $p$ and complexity $\bar{k}$ is

$$
\begin{aligned}
\pi_{i}(p, \bar{k})= & p \int_{p}^{1} \ldots \int_{p}^{1}\left[\sum_{m=0}^{n-1} \mathrm{P}_{n-1}^{m}(1-\gamma(n-m))\right] d F\left(p_{j}\right) \ldots d F\left(p_{l}\right)+ \\
& \frac{p}{n} \int_{p_{0}}^{1} \ldots \int_{p_{0}}^{1}\left[\sum_{m=0}^{n-1} \mathrm{P}_{n-1}^{m} \gamma(n-m)\right] d F\left(p_{j}\right) \ldots d F\left(p_{l}\right) .
\end{aligned}
$$

The expression for $\pi_{i}(p, \bar{k})$ is analogous to the one for $\pi_{i}(p, \underline{k})$, with the difference that, when exactly $m \geq 0$ out of $n-1$ rivals use $\underline{k}$, as firm $i$ employs $\bar{k}$, there are $\gamma(n-m)$ confused consumers and $(1-\gamma(n-m))$ experts.

The incremental profitability of an increase in complexity $\left(\pi_{i}(p, \bar{k})-\pi_{i}(p, \underline{k})\right)$ is given by

$$
\begin{gathered}
-\frac{(n-1) p}{n} \int_{p}^{1} \ldots \int_{p}^{1}\left[\sum_{m=0}^{n-1} \mathrm{P}_{n-1}^{m}(\gamma(n-m)-\gamma(n-m-1))\right] d F\left(p_{j}\right) \ldots d F\left(p_{k}\right) \\
+\frac{p}{n} \int_{p_{0}}^{p} \ldots \int_{p_{0}}^{p}\left[\sum_{m=0}^{n-1} \mathrm{P}_{n-1}^{m}(\gamma(n-m)-\gamma(n-m-1))\right] d F\left(p_{j}\right) \ldots d F\left(p_{k}\right) .
\end{gathered}
$$

This expression can be simplified using the following result which draws on (3). 
Lemma 4 Suppose $n \geq 2$ and $m \leq n-1$. Let $\mathbf{p}_{-i}$ be the ex post price profile of firm $i$ 's competitors and $\Lambda\left(p_{j}\right) \equiv\left[1+\left(1-\lambda\left(p_{j}\right)\right)(\beta-1)\right]$, where $p_{j}$ is an element of $\mathbf{p}_{-i}$. Then,

$$
\sum_{m=0}^{n-1} \mathbf{P}_{n-1}^{m}(\gamma(n-m)-\gamma(n-m-1))=\gamma(1) \prod_{j \neq i} \Lambda\left(p_{j}\right) .
$$

By (4), the incremental profitability of an increase in complexity becomes

$$
\pi_{i}(p, \bar{k})-\pi_{i}(p, \underline{k})=p \gamma(1)\left(-\frac{n-1}{n} \prod_{j \neq i} \int_{p}^{1} \Lambda\left(p_{j}\right) d F\left(p_{j}\right)+\frac{1}{n} \prod_{j \neq i} \int_{p_{0}}^{p} \Lambda\left(p_{j}\right) d F\left(p_{j}\right)\right) .
$$

This expression generalizes the incremental profitability of an increase in complexity presented in section 4 , and similar reasoning leads to the next result.

Proposition 2 In symmetric mixed-strategy oligopoly equilibrium, a firm's complexity level depends only on its price. The firms choose prices according to a c.d.f. $F(p)$ with support $S=\left[p_{0}, 1\right]$. If $p<\hat{p}$ the firms choose complexity $\underline{k}$, if $p>\hat{p}$ the firms choose complexity $\bar{k}$, and if $p=\hat{p}$ the firms are indifferent between the two complexity levels.

When a firm chooses relatively low prices, it benefits from more market transparency, as it is more likely to serve the experts. In contrast, when a firm chooses relatively high prices, it relies more on confused consumers and benefits from higher complexity. As a result, in equilibrium there is a positive relationship between prices and price complexity levels and a firm's complexity choice is determined by its price draw. Each firm assigns probability $\lambda=F(\hat{p}) \in(0,1)$ to complexity level $\underline{k}$ and $1-\lambda$ to complexity level $\bar{k}$.

The proof of the next result uses the approach illustrated in section 4 and is relegated to the appendix. Drawing on Proposition 2, a firm's expected profit for a price $p \in\left[p_{0}, \hat{p}\right.$ ) (which is associated with $\underline{k}$ ) and for a price $p \in[\hat{p}, 1]$ (which is associated with $\bar{k}$ ), and mixed strategy equilibrium constant profit conditions are also presented there. Recall that $\gamma(n)=\gamma_{\max }$.

Proposition 3 For $n \geq 2$ and $\beta \neq 1$, there is a unique symmetric equilibrium, where the firms choose prices randomly according to a c.d.f. $F(p)$ defined on $\left[p_{0}, 1\right]$ where $p_{0}>0$. Each firm chooses complexity $\bar{k}$ with probability $1-\lambda=1-F(\hat{p})$, where

$$
1-\lambda=\frac{n^{-1 /(n-1)}}{1+(\beta-1)\left(1-n^{-1 /(n-1)}\right)} \in(0,1),
$$

and complexity $\underline{k}$ with probability $\lambda$. Each firm's expected profit is

$$
\pi=\gamma_{\max } \frac{\left[n(1-\lambda)^{n-1} \beta^{n}-1\right]}{n\left(\beta^{n}-1\right)} .
$$

If $\gamma_{\max }<1$, then $\hat{p}<1$ and $F(p)$ is continuous everywhere. If $\gamma_{\max }=1$, then $\hat{p}=1$ and $F(p)$ is continuous for $p \in\left[p_{0}, \hat{p}\right)$, but has an atom at $\hat{p}$.

In homogeneous product markets, where firms compete by simultaneously choosing prices and price complexity levels, there is dispersion in both dimensions in equilibrium. These findings are consistent with observed patters in markets for financial and banking products, or markets for gas 
and electricity. Firms make strictly positive profits and charge prices in excess of marginal cost. The firms' equilibrium strategies and market outcomes depend on market structure (as captured by $n$ ), the curvature of the confusion technology $(\beta)$, and the degree of consumer sophistication $\left(\gamma_{\max }\right)$.

\section{A Model without Second Order Effects}

So far, the focus has been on $\beta \neq 1$. Below, a variant of the model where $\beta=1$ (and so the confusion technology is linear) is discussed. The preliminary results in Lemmas 1-3 carry over unchanged. The $\beta=1$ case is closely related to the analysis in Carlin (2009). There, the firms choose complexity levels from a closed interval $[\underline{k}, \bar{k}]$, but in symmetric mixed-strategy equilibrium they randomize only between the extreme values of this interval. His analysis proves the existence of a cut-off mixed strategy equilibrium. The next result fully characterizes the unique symmetric equilibrium of a variant with binary price complexity levels $\left(k_{i} \in\{\underline{k}, \bar{k}\}\right)$. The proof is relegated to the online appendix.

Proposition 4 For $n \geq 2$ and $\beta=1$, there is a unique symmetric equilibrium, where firms choose prices randomly according to a c.d.f. $F(p)$ defined on $\left[p_{0}, 1\right]$ where $p_{0}>0$. Each firm chooses complexity $\bar{k}$ with probability $1-\lambda=1-F(\hat{p})$, given by

$$
1-\lambda=n^{-\frac{1}{n-1}} \in(0,1)
$$

and complexity $\underline{k}$ with probability $\lambda$. Each firm's equilibrium profit $\pi$ is given by

$$
\pi=\gamma_{\max } \frac{1-n^{-\frac{1}{n-1}}+n^{\frac{n-2}{n-1}}}{n^{2}} .
$$

If $\gamma_{\max }<1$, then $\hat{p}<1$ and $F(p)$ is continuous everywhere. If $\gamma_{\max }=1$, then $\hat{p}=1$ and $F(p)$ is continuous for $p \in\left[p_{0}, \hat{p}\right)$ but has an atom at $\hat{p}$.

\section{Discussion and Policy Implications}

This section explores equilibrium comparative statics and convergence results, and discusses their implications for competition and consumer protection policy. Building on Propositions 3 and 4, it focuses on the impact of changes in the number of firms $(n)$, the degree of consumer sophistication $\left(\gamma_{\max }\right)$, and the concavity or convexity of the confusion technology $(\beta)$ on the equilibrium probability of using low complexity, the expected share of confused consumers, and the expected industry profit.

\subsection{Probability of Using Low Complexity}

Corollary 3 In the symmetric mixed-strategy equilibrium, the probability that each firm assigns to the low complexity level $\underline{k}(\lambda)$ decreases in the number of firms ( $n)$, is independent of the degree of consumer sophistication $\left(\gamma_{\max }\right)$, and increases in the degree of convexity $\beta$. Moreover, $\lim _{n \rightarrow \infty} \lambda=0$. 
Consistent with existing results, as the number of competitors increases, firms rely more on consumer confusion and so choose high price complexity more frequently. In the limit, as the number of competitors goes to infinity, the firms use high price complexity almost surely. Hence, in settings where firms compete by choosing both prices and price complexity, an increase in the number of firms leads to an increase in the overall complexity of the market. Like in our duopoly framework, the frequency with which firms use low complexity is independent of consumers' degree of sophistication. So, we identify the possibility that initiatives which improve consumer awareness may not lead to reductions in the overall market complexity.

For a given number of firms, a lower degree of concavity or higher degree of convexity of the confusion technology leads to a decrease in market complexity. When $\beta \in(0,1)$, higher complexity of rivals' offers reduces the increase in confusion resulting from a firm's incremental complexity. However, when $\beta$ increases, this reduction gets smaller and $\lambda$ increases because a firm's incentives to choose high complexity are reduced. When $\beta>1$, higher rival complexity magnifies the increase in confusion resulting from the incremental complexity of a firm's offer and an increase in $\beta$ further weakens a firm's incentives to use high complexity. Figure 3 provides an illustration.

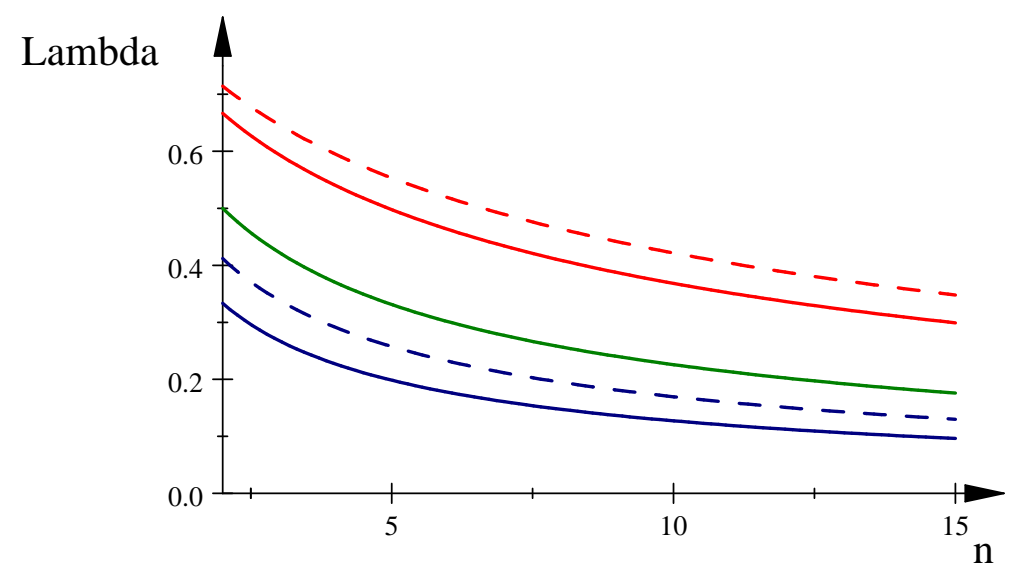

Figure 3: Equilibrium probability of using $\underline{k}(\lambda)$ as a function of $n$. From bottom to top for $\beta$ equal to $0.5,0.7,1,2$, and 2.5 .

\subsection{Expected Share of Confused Consumers}

The equilibrium expected share of confused consumers is

$$
\exp [\gamma] \equiv \sum_{m=0}^{n} C_{n}^{m} \lambda^{m}(1-\lambda)^{n-m} \gamma(n-m)
$$

For a given number of firms, $\exp [\gamma]$ aggregates the shares of confused consumers in the sequence $(\gamma(n-m))_{m=0}^{n}$, weighting each share by the overall probability that $m$ firms choose low complexity and $n-m$ firms choose high complexity. Using (3) for $\beta \neq 1$ and (4) for $\beta=1$, the equilibrium 
expected share of confused consumers becomes

$$
\exp [\gamma]=\left\{\begin{array}{c}
\gamma_{\max } \frac{[1+(1-\lambda)(\beta-1)]^{n}-1}{\beta^{n}-1} \text { for } \beta \neq 1 \\
\gamma_{\max }(1-\lambda) \text { for } \beta=1
\end{array} .\right.
$$

To focus on the impact of the confusion technology's curvature on market outcomes, the comparative statics and convergence results below assume that the upper bound of the share of confused consumers is independent of the number of firms, i.e. $\gamma(n)=\gamma_{\max }$ is a constant. By fixing the maximal share of confused consumers, when the number of firms is increased, each firm is made smaller with respect to the market, as its maximal base of confused consumers is $\gamma_{\max } / n .{ }^{15}$

The convergence properties of the expected share of confused consumers with respect to the number of firms depend on whether the confusion technology is concave or convex in firms' complexity choices.

Corollary 4 In the symmetric mixed-strategy equilibrium, the expected share of confused consumers in (19) increases in $\gamma_{\max }$ and

$$
\lim _{n \rightarrow \infty} \exp [\gamma]=\left\{\begin{array}{rl}
\gamma_{\max } & \text { for } \beta \in(0,1] \\
0 & \text { for } \beta>1
\end{array} .\right.
$$

If the confusion technology is convex, the expected share of confused consumers converges to zero as the number of firms goes to infinity. For simplicity, this analysis assumes that $\gamma(0)=0$ (i.e., when all firms use low complexity, there are no confused consumers). If $\gamma(0)>0$ instead (i.e., if some consumers get confused even when all firms use $\underline{k}$ ), then with convex confusion technology, the expected share of confused consumers would converge instead to the minimal level, $\gamma(0)$; see footnote 2. In contrast, if the confusion technology is concave $(\beta \in(0,1))$, the expected share of confused consumers is bounded away from zero and converges to $\gamma_{\max }$ as the number of firms goes to infinity.

The curvature of the confusion technology in this model is captured by the sequence of shares $(\gamma(n-m))_{m=0}^{n}$. A simple illustration is provided below.

Example 2 Consider (3). If $\beta=2$, then $\gamma(n-m)=\gamma_{\max }\left(2^{n-m}-1\right) /\left(2^{n}-1\right)$ and $\lim _{n \rightarrow \infty} \gamma(n-$ $m)=\gamma_{\max } 2^{-m}$. If $\beta=1 / 2$, then $\gamma(n-m)=\gamma_{\max }\left(0.5^{n-m}-1\right) /\left(0.5^{n}-1\right)$ and $\lim _{n \rightarrow \infty} \gamma(n-m)=$ $\gamma_{\max }$.

In relatively large oligopoly markets, convexity requires a relatively ineffective confusion technology and, although the firms use almost surely high complexity, the expected share of confused consumers converges to zero. In contrast, concavity requires a relatively effective confusion technology. In nearly competitive markets with concave confusion technology, as the firms use almost surely high complexity, the expected share of confused consumers is close to the highest level. This is also the case with linear confusion technology, so the results for $\beta=1$ are qualitatively similar to those that obtain under concavity.

\footnotetext{
${ }^{15}$ For a discussion of comparative statics in large oligopoly markets, see Chapter 5.2.5. in Vives (2001).
} 
Beyond the convergence results presented in Corollary 4, numerical simulations indicate that the expected number of confused consumers, $\exp [\gamma]$, increases monotonically in $n$ for $\beta \in(0,1]$. For $\beta=1$, this monotonicity result can be derived analytically using Corollary 3 . For all $\beta>1$, $\exp [\gamma]$ decreases in $n$ at least when $n$ is large enough. For $\beta \geq 2$, its value at $n=2$ is larger than the value at $n=3$, and then $\exp [\gamma]$ decreases monotonically in $n$ for $n \geq 3 .{ }^{16}$ However, for $\beta \in(1,1.8)$ it peaks at some value $n_{0}(\beta) \geq 3$. Numerical examples also indicate that the expected share of confused consumers strictly decreases in $\beta$ for given $n$ and increases in $\gamma_{\max }$.

Example 3 Let $\gamma_{\max }=1$. Consider $\beta=1 / 2$, 1, or 2 . The expected share of confused consumers as a function of the number of firms is illustrated in Figure 4 and presented in the appendix.

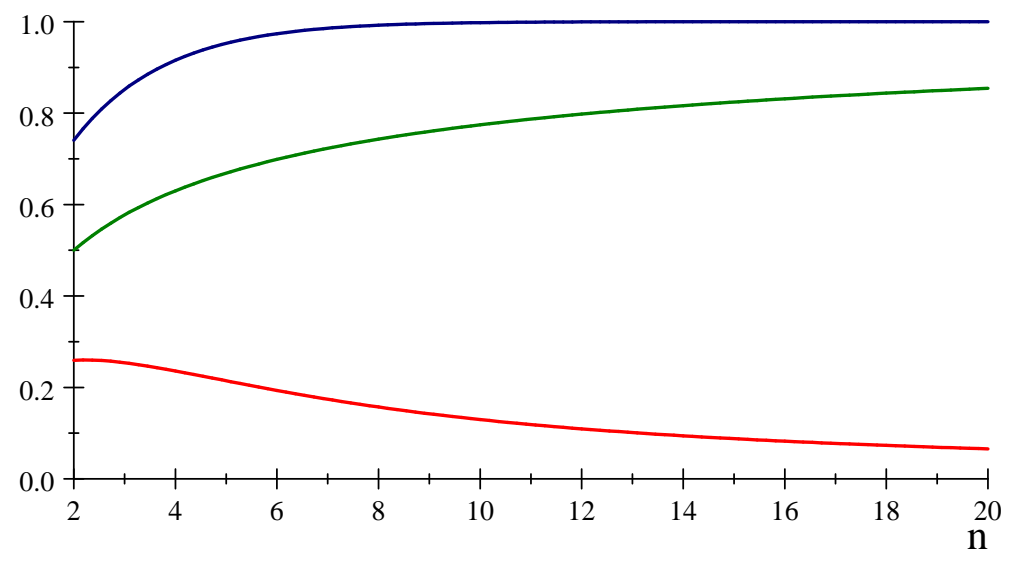

Figure 4: Expected share of confused consumers in equilibrium for $\gamma_{\max }=1$. From bottom to top for $\beta=2, \beta=1$, and $\beta=1 / 2$.

As our analysis holds the maximal share of confused consumers fixed, the number of firms does not have a direct impact on consumer sophistication (as measured by $\gamma_{\max }$ ). Suppose instead that the maximal share of confused consumers $\left(\gamma_{\max }=\gamma(n)\right)$ is an increasing function of the number of firms $(n)$. This could be the case in a market where there is choice overload and consumers are more likely to make random choices when they face more options. Using (19), it is easy to see that the qualitative results in Corollary 4 carry over unchanged. For $\beta>1, \gamma(n)$ is bounded above by $\gamma_{\max }$, so that in the limit, the expected share of confused consumers still goes to zero. For $\beta \in(0,1]$, the expected share of confused consumers converges to $\lim _{n \rightarrow \infty} \gamma(n)>\gamma(2)>0$.

Now, suppose that $\gamma(n)$ is a decreasing function of $n$, that is, the larger the number of firms, the higher the minimal share of experts is. Arguably, this is a less realistic case as it requires the number of firms in the market to have a positive impact on consumer sophistication as measured by the maximal share of confused consumers. For $\beta>1, \gamma(n)$ is bounded below by zero, so the expected share of confused consumers still converges to zero. However, for $\beta \in(0,1]$, the expected share of confused consumers may converge to zero as almost all consumers are sophisticated in

\footnotetext{
${ }^{16}$ For $\beta \in[2,2.15)$, the expected number of confused is an inverted $\mathrm{U}$ function of $n$, but reaches a maximum at some $n_{0}(\beta) \in(2,3)$.
} 
nearly competitive markets. See also the examples in Carlin, 2009 (section 4, p. 284): in the first one, like in this analysis, the maximal share of confused consumers is independent of $n$, while in the second it decreases with $n$.

\subsection{Expected Industry Profit}

Corollary 5 In symmetric mixed-strategy equilibrium, expected industry profit $\left(\pi_{n}=n \pi\right)$ increases in $\gamma_{\max }$ and is strictly larger than expected share of confused consumers. Furthermore,

$$
\lim _{n \rightarrow \infty} \pi_{n}=\left\{\begin{array}{rl}
\gamma_{\max } & \text { for } \beta \in(0,1] \\
0 & \text { for } \beta>1
\end{array} .\right.
$$

An increase in the level of consumer sophistication has a positive effect on expected consumer surplus (as total welfare is normalized to one). Note that in this model consumer surplus is only determined by equilibrium prices. In practice price complexity might directly affect consumers, for instance, by raising their information gathering or processing costs. This would be the case, for instance, if the experts require more time to identify the lowest price when complexity is higher. Combined with the findings in Corollary 3, this implies that an improvement in consumer awareness boosts consumer surplus, but this may not be reflected by a reduction in overall market complexity. Hence, the insight from the duopoly model that market transparency may not be a good indicator of market performance carries over to oligopoly settings.

In nearly perfectly competitive markets, when the confusion technology is concave, or when it is linear, expected industry profit is bounded away from zero. As expected total profit is larger than the expected share of confused consumers, this result is closely related to Corollary 4. On the other hand, when the confusion technology is convex, expected profit converges to zero as the number of firms converges to infinity. In this case, although firms choose high complexity almost surely, the confusion technology is relatively inefficient and markets with many firms may be highly competitive.

In general, industry profit and implicitly consumer surplus are not monotonic in the number of firms. An increase in the number of competitors gives firms stronger incentives to undercut and attract the experts, but also to use more frequently high complexity $(\bar{k})$, which is associated with relatively higher prices. Numerical simulations also suggest that industry profit decreases in $\beta$ for given $\gamma_{\max }$ and $n$. Figure 5 provides an illustration for $\gamma_{\max }=1$ and Table 1 presents equilibrium outcomes for various values of $n$ and $\beta$, when $\gamma_{\max }=0.4$.

In the table, $p_{0}$ decreases in $n$ for given $\beta$ and in $\beta$ for given $n$, whereas $\hat{p}$ decreases in $n$ for $\beta=1 / 2$, it increases in $n$ for $\beta=1$ and $\beta=2$, and it increases in $\beta$ for given $n$. Expected industry profit and the expected share of confused consumers increase in $n$ for $\beta=1 / 2$ and $\beta=1$, decrease 


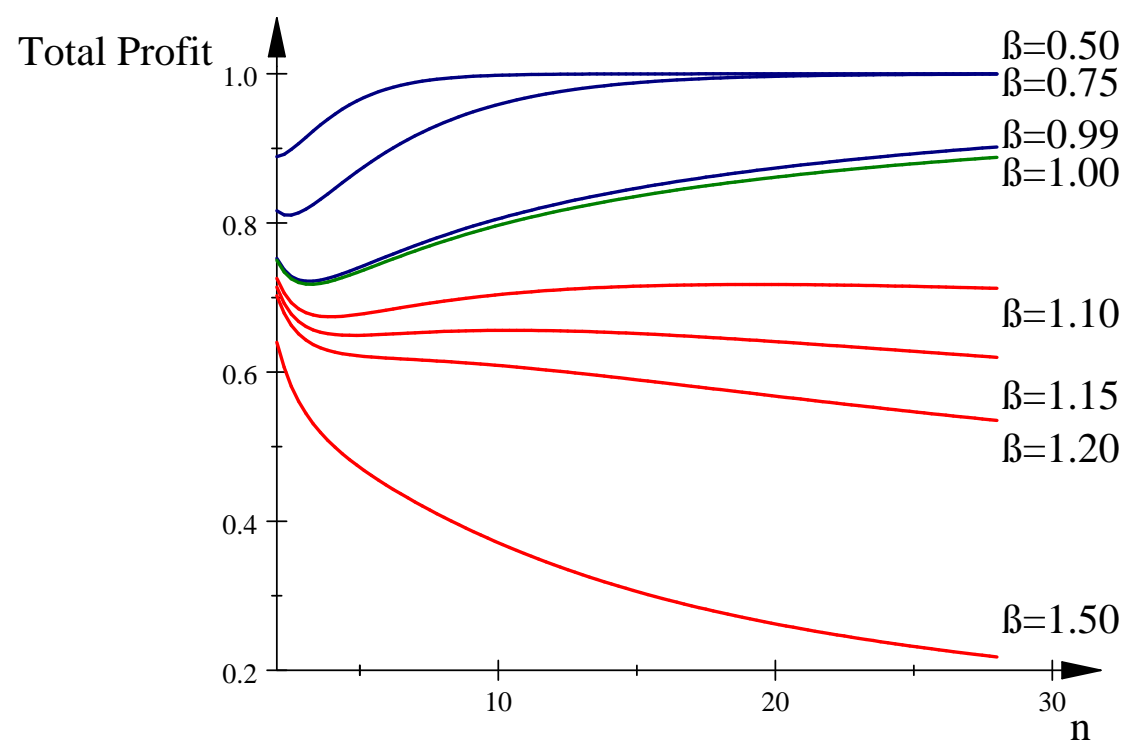

Figure 5: Equilibrium industry profit $\left(\pi_{n}\right)$ as a function of $n$ for $\gamma_{\max }=1$ and values of $\beta$.

in $n$ for $\beta=2$, and decreases in $\beta$ for given $n$.

\begin{tabular}{|l|l|l|l|l|l|l|l|}
\hline \multicolumn{7}{|c|}{ Table $1\left(\gamma_{\max }=0.4\right)$} \\
\hline$\beta$ & $n$ & $\lambda$ & $\pi$ & $\pi_{n}$ & $p_{0}$ & $\hat{p}$ & $\exp [\gamma]$ \\
\hline $1 / 2$ & 3 & 0.268 & 0.122 & 0.365 & 0.149 & 0.275 & 0.852 \\
\hline $1 / 2$ & 4 & 0.227 & 0.094 & 0.377 & 0.125 & 0.254 & 0.916 \\
\hline $1 / 2$ & 10 & 0.127 & 0.04 & 0.4 & 0.062 & 0.185 & 0.998 \\
\hline 1 & 3 & 0.423 & 0.096 & 0.287 & 0.107 & 0.324 & 0.578 \\
\hline 1 & 4 & 0.370 & 0.072 & 0.289 & 0.084 & 0.325 & 0.63 \\
\hline 1 & 10 & 0.226 & 0.032 & 0.320 & 0.043 & 0.347 & 0.774 \\
\hline 2 & 3 & 0.594 & 0.056 & 0.169 & 0.058 & 0.363 & 0.254 \\
\hline 2 & 4 & 0.540 & 0.034 & 0.14 & 0.036 & 0.374 & 0.236 \\
\hline 2 & 10 & 0.368 & 0.006 & 0.064 & 0.007 & 0.399 & 0.13 \\
\hline
\end{tabular}

In a discussion of models where framing limits the comparability of competing offers, Spiegler (2016) introduces a property called 'enforceable comparability' (EC). ${ }^{17}$ When EC is satisfied, industry profit is independent of the number of firms. However, in settings where a unilateral increase in the price format (or complexity level) leads to more consumer confusion irrespective of the rivals' choices, EC is not satisfied. This is the case here, in Carlin (2009), and in parts of the analysis in Chioveanu and Zhou (2013). The latter analysis identifies the possibility that industry profit may be non-monotonic in the number of firms. The current analysis contributes

\footnotetext{
${ }^{17}$ Intuitively, 'enforceable comparability' is satisfied if there exists a probability distribution over the set of formats which equalizes a firm's expected share of confused consumers across all frame choices for any frame profile of the rivals.
} 
by providing a novel taxonomy of the impact of the number of firms on industry profits when EC fails. In particular, it shows that in a Carlin-type setting the curvature of the confusion technology drives the convergence properties of expected industry profit in large markets.

Our results may provide policy guidance by distinguishing when a focus on the number of firms is desirable in large markets. From an empirical perspective, the confusion technology can be approximated by using information on the degree of market complexity and on the share of consumers who make suboptimal choices for a given number of firms. Recent analyses of retail financial markets propose different measures of complexity, some of them related to the length of product descriptions (e.g. number of pages or characters) or the number of technical terms (e.g., words that appear in the glossary); see, for instance, Célérier and Vallée (2017) and Ghent, Torous, and Valkanov (2017). Using survey and consumer-level data from liberalized retail utility markets, a fast-growing stream of empirical work develops methods to estimate preferences in markets with search frictions and finds evidence of consumer inertia and confusion; see, for example, Hortaçsu, Madanizadeh, and Puller (2017) and the references therein.

In practice, consumer protection intervention or market developments might influence the patterns of consumer behavior and, implicitly, the characteristics of the confusion technology. For instance, price format standardization might facilitate consumer learning by doing and have an impact on the curvature of the confusion technology. Online technologies which facilitate information sharing in markets may have similar effects. Our results suggest that such interventions or developments affect market performance and may have to be taken into consideration in the design of competition policy and in the assessment of its interaction with consumer policy.

\section{Conclusions}

This paper analyzes a homogeneous product market where firms compete by choosing both prices and price complexity levels. Complexity makes it harder for consumers to assess prices and identify the best offer, and is a source of consumer confusion. A distinctive feature of the proposed model is that it accommodates more general confusion technologies and, implicitly, consumer behaviors. Our analysis distinguishes between two properties of the confusion technology, its curvature (concavity or convexity) with respect to the overall complexity and the degree of consumer sophistication, and isolates their comparative statics effects. To separate these effects and develop a tractable framework, the oligopoly model parametrizes the curvature of the confusion technology.

Our results suggest that confusion technology characteristics have an impact on market outcomes and implications for competition and consumer protection policy. An increase in the number of competitors leads to an increase in market complexity but, in relatively small markets, it has ambiguous effects on consumer surplus. In large markets, firms use high price complexity almost surely and, if the confusion technology is convex, expected industry profits converge to zero. In large markets with concave or linear confusion technology, expected industry profits converge to 
the highest level. An improvement in consumer sophistication boosts consumer surplus but may not reduce overall market complexity. Therefore, the effectiveness of interventions which increase consumer awareness and boost their surplus might not be reflected in the degree of market transparency they deliver.

\section{Appendix}

\subsection{Preliminary Results}

Proof of Lemma 3. (i) Let $\widetilde{S}$ be the convex hull of $S$. Suppose there is a gap $G \subset \widetilde{S}$. Let $A=\{p \in S \mid p \leq \inf G\}, p_{a}=\max A$ and $p^{\prime}=\sup G$. Clearly, $p_{a} \in S$ and $F\left(p_{a}\right)=F\left(p^{\prime}\right)$. But then $\pi_{i}\left(p^{\prime}, k, \xi_{j}\right)>\pi_{i}\left(p_{a}, k, \xi_{j}\right)$. A contradiction.

(ii) (a) Suppose there is a mass point at some $p^{\prime} \in S$ with $0<p^{\prime}<\max S$. Then, there is a positive probability of a tie at this price. (a1) If $\lambda\left(p^{\prime}\right)>0$, the expected share of experts at this price is strictly positive when all firms tie. Then, firm $i$ is better off deviating to $p^{\prime}-\epsilon$ as there is a discrete increase in market share and only a marginal decrease in price. This deviation applies also at $p^{\prime}=\max S$, so that firms cannot have a mass point at the upper bound of $S$. (a2) If $\lambda\left(p^{\prime}\right)=0$, the share of confused consumers at this price is $\gamma_{\max }$. If $\gamma_{\max }<1$, the argument in (a1) applies unchanged. If $\gamma_{\max }=1$, in the event of a tie at $p^{\prime}$, all consumers are confused and firm $i$ is better off moving mass from price $p^{\prime}$ to price $p=\max S$. Note that this argument does not rule out a mass point at the upper bound of $S$ when $\gamma_{\max }=1$. (c) Suppose there is a mass point at $p^{\prime}=0$. In this case, all firms make zero profits. If $\lambda\left(p^{\prime}\right)<1$, then there are some confused consumers when firms tie at this price and firm $i$ is better off deviating to $p=1$ and making positive profits on its share of confused consumers. If $\lambda\left(p^{\prime}\right)=0$, all consumers are experts when firms tie, but then firm $i$ is better off deviating to $p=1$ and $k_{i}=\bar{k}$, where it makes positive profit.

(iii) It follows from (i) that in symmetric equilibrium $S$ is a bounded interval. Suppose $p_{h}=$ $\sup S<1$. If $\gamma_{\max }<1$, by (ii) firms pricing c.d.f.s are continuous everywhere, so a firm charging $p \rightarrow p_{h}$ sells only to its share of confused consumers and it is clearly better off deviating to a higher price $p=1$. If $\gamma_{\max }=1$, there may be a positive probability of a tie at $p_{h}$ but, as all consumers are confused when all firms tie, deviating to $p=1$ is profitable. A contradiction. As $p_{h}=1$, firms' expected profit in the mixed strategy equilibrium will be strictly positive, so it must be that $p_{0}=\min S>0$.

\subsection{Duopoly Analysis}

\section{Expected Profits}

Using (8), we re-write a firm's expected profits. If firm $i$ chooses complexity $\underline{k}$ and charges a price $p \in\left[p_{0}, \hat{p}\right)$, as $\gamma(0)=0$, (5) becomes

$$
\pi_{i}(p, \underline{k})=p\left[(\lambda-F(p))+(1-\lambda)(1-\gamma(1))+\frac{(1-\lambda) \gamma(1)}{2}\right] .
$$


If instead firm $i$ chooses $\bar{k}$ and charges $p \in[\hat{p}, 1]$, expression (6) becomes

$$
\pi_{i}(p, \bar{k})=p\left[(1-F(p))(1-\gamma(2))+\frac{\lambda \gamma(1)}{2}+\frac{(1-\lambda) \gamma(2)}{2}\right] .
$$

\section{Cumulative Price Distributions}

The symmetric equilibrium price distribution is presented below.

$$
1-F(p)=\left\{\begin{array}{c}
\frac{\gamma_{\max }}{2(\beta+1)^{2}}\left(\frac{2 \beta+1}{p}+1\right) \text { for } p \in\left[p_{0}, \hat{p}\right) \\
\frac{\gamma_{\max }(2 \beta+1)}{2(\beta+1)^{2}\left(1-\gamma_{\max }\right)}\left(\frac{1}{p}-1\right) \text { for } p \in[\hat{p}, 1], \text { if } \gamma_{\max }<1
\end{array}\right.
$$

Proof of Proposition 1. It is easy to see that $\lambda, p_{0}, \hat{p}$, and $F(p)$ as presented in (9), (10), and (A1) are all well defined.

Consider a unilateral deviation to $\left(p<p_{0}, \underline{k}\right)$. The deviator's market share is equal to that corresponding to $p=p_{0}$ but, as the price is lower, this deviation is not profitable.

Consider a unilateral deviation to $(p>\hat{p}, \underline{k})$. This results in deviation profit

$\pi^{d}(p, \underline{k})=p\left[(1-F(p))(1-\gamma(1))+\frac{\lambda \gamma(0)}{2}+\frac{(1-\lambda) \gamma(1)}{2}\right]<$

$\pi(p, \bar{k})=p\left[(1-F(p))(1-\gamma(2))+\frac{\lambda \gamma(1)}{2}+\frac{(1-\lambda) \gamma(2)}{2}\right]$.

The inequality follows as, for $p>\hat{p}, \frac{\left(\pi^{d}(p, \underline{k})-\pi(p, \bar{k})\right)}{p}=$

$\left[(1-F(p))(\gamma(2)-\gamma(1))-\frac{\lambda(\gamma(1)-\gamma(0))}{2}-\frac{(1-\lambda)(\gamma(2)-\gamma(1))}{2}\right]=$

$(\gamma(2)-\gamma(1))\left[(1-F(p))-\frac{\lambda}{2 \beta}-\frac{(1-\lambda)}{2}\right]<$

$(\gamma(2)-\gamma(1))\left[(1-F(\hat{p}))-\frac{\lambda}{2 \beta}-\frac{(1-\lambda)}{2}\right]=0$.

Consider a unilateral deviation to $(p<\hat{p}, \bar{k})$. This results in deviation profit

$\pi^{d}(p, \bar{k})=p\left[(1-\lambda)(1-\gamma(2))+(\lambda-F(p))(1-\gamma(1))+\frac{(1-\lambda) \gamma(2)}{2}+\frac{\lambda \gamma(1)}{2}\right]<$

$\pi(p, \underline{k})=p\left[(1-\lambda)(1-\gamma(1))+(\lambda-F(p))+\frac{(1-\lambda) \gamma(1)}{2}\right]$.

The inequality follows as, for $p<\hat{p}, \frac{\left(\pi^{d}(p, \bar{k})-\pi(p, \underline{k})\right)}{p}=$

$-(1-\lambda)(\gamma(2)-\gamma(1))-(\lambda-F(p)) \gamma(1)+\frac{(1-\lambda)(\gamma(2)-\gamma(1))}{2}+\frac{\lambda \gamma(1)}{2}=$

$(\gamma(2)-\gamma(1)) \frac{-2(\lambda-F(p))-\beta(1-\lambda)+\lambda}{2 \beta}<(\gamma(2)-\gamma(1)) \frac{-\beta(1-\lambda)+\lambda}{2 \beta}=0$.

Equilibrium Expected Share of Confused Consumers.

$$
\sum_{m=0}^{2} C_{2}^{m} \lambda^{m}(1-\lambda)^{2-m} \gamma(2-m)=\frac{\gamma_{\max }(3 \beta+1)}{(\beta+1)^{3}} .
$$

For $\gamma_{\max }>0$ is strictly lower than expected industry profits. 
Proof of Corollary 1. Using (9), $d \lambda / d \beta=1 /(\beta+1)^{2}>0$.

Using (10), $d p_{0} / d \beta=-2 \gamma_{\max }\left[\gamma_{\max }+2(\beta+1) \beta\right] /\left(2(\beta+1)^{2}-\gamma_{\max }\right)^{2}<0$.

Using (10), $d \hat{p} / d \beta=2 \gamma_{\max }\left(1-\gamma_{\max }\right) /\left[2(\beta+1)-\gamma_{\max }\right]^{2} \geq 0$, with equality iff $\gamma_{\max }=1$.

Using (9), $d \pi / d \beta=(1 / 2)\left(d \pi_{2} / d \beta\right)=-\gamma_{\max } \beta /(\beta+1)^{3}<0$.

Differentiating (A2) w.r.t. $\beta$ gives $-6 \gamma_{\max } \beta /(\beta+1)^{4}<0$.

Using (A1), $E(p)=\int_{p_{0}}^{\hat{p}}(1-F(p)) d p+\int_{\hat{p}}^{1}(1-F(p)) d p+p_{0}$. By Leibniz's rule,

$d E(p) / d \beta=(1-F(\hat{p}))(d \hat{p} / d \beta)-\left(1-F\left(p_{0}\right)\right) d p_{0} / d \beta+$

$\int_{p_{0}}^{\hat{p}}(-d F(p) / d \beta) d p-(1-F(\hat{p}))(d \hat{p} / d \beta)+\int_{\hat{p}}^{1}(-d F(p) / d \beta) d p+d p_{0} / d \beta=$

$\int_{p_{0}}^{\hat{p}}(-d F(p) / d \beta) d p+\int_{\hat{p}}^{1}(-d F(p) / d \beta) d p$.

But, for $p \in\left[p_{0}, \hat{p}\right)$, using $(\mathrm{A} 1), d F(p) / d \beta=\gamma_{\max }(\beta+p) /(\beta+1)^{3} p>0$. For $p \in[\hat{p}, 1]$ and $\gamma_{\max }<1$, $d F(p) / d \beta=\gamma_{\max } \beta(1-p) /(\beta+1)^{3}\left(1-\gamma_{\max }\right) p>0$. Then it follows that $d E(p) / d \beta<0$. For $\gamma_{\max }=$ $1, \hat{p}=1$ and the second integral in $E(p)$ is a constant, so $d E(p) / d \beta=\int_{p_{0}}^{\hat{p}}(-d F(p) / d \beta) d p<0$.

Conditional Price Distribution. The price distribution conditional on the price being below the cut-off value $\hat{p}$ and the corresponding conditional expected price are given by

$$
\begin{aligned}
& F(p \quad \mid \quad p<\hat{p})=F(p) / F(\hat{p})=\frac{2(\beta+1)^{2}-\gamma_{\max }}{2(\beta+1) \beta}-\frac{\gamma_{\max }(2 \beta+1)}{2(\beta+1) \beta p} \\
& E(p \quad \mid \quad p<\hat{p})=\frac{\gamma_{\max }(2 \beta+1)}{2(\beta+1) \beta} \ln \frac{2(\beta+1)^{2}-\gamma_{\max }}{2(\beta+1)-\gamma_{\max }}
\end{aligned}
$$

Proof of Corollary 2. Using (9), $d \lambda / d \gamma_{\max }=0$.

Using (10), $d p_{0} / d \gamma_{\max }=2(2 \beta+1)(\beta+1)^{2} /\left[2(\beta+1)^{2}-\gamma_{\max }\right]^{2}>0$.

Using (10), $d \hat{p} / d \gamma_{\max }=2(2 \beta+1)(\beta+1) /\left[2(\beta+1)-\gamma_{\max }\right]^{2}>0$.

Using (9), $d \pi / d \gamma_{\max }=(2 \beta+1) / 2(\beta+1)^{2}>0$.

Differentiating (A2) w.r.t. $\gamma_{\max }$ gives $-(3 \beta+1) /(\beta+1)^{3}<0$.

Using (A1), $E(p)=\int_{p_{0}}^{\hat{p}}(1-F(p)) d p+\int_{\hat{p}}^{1}(1-F(p)) d p+p_{0}$. Then, by Leibniz's rule,

$\int_{p_{0}}^{\hat{p}}\left(-d F(p) / d \gamma_{\max }\right) d p+\int_{\hat{p}}^{1}\left(-d F(p) / d \gamma_{\max }\right) d p$.

But, for $p \in\left[p_{0}, \hat{p}\right)$, using $(\mathrm{A} 1), d F(p) / d \gamma_{\max }=-[(2 \beta+1) / p+1] / 2(\beta+1)^{2}<0$. For $p \in$ $[\hat{p}, 1]$ and $\gamma_{\max }<1, d F(p) / d \gamma_{\max }=-(2 \beta+1)(1-p) / 2(\beta+1)^{2} \gamma_{\max } p<0$. It follows that $d E(p) / d \gamma_{\max }>0$. For $\gamma_{\max }=1, \hat{p}=1$ and the second integral in $E(p)$ is a constant, so $d E(p) / d \gamma_{\max }=\int_{p_{0}}^{\hat{p}}\left(-d F(p) / d \gamma_{\max }\right) d p>0$.

Using (A3), it follows that

$d E(p \mid p<\hat{p}) / d \gamma_{\max }=$

$\left\{\frac{\gamma_{\max }(2 \beta+1)}{\left[2(\beta+1)-\gamma_{\max }\right]\left[2(\beta+1)^{2}-\gamma_{\max }\right]}+\frac{2 \beta+1}{2(\beta+1) \beta} \ln \frac{2(\beta+1)^{2}-\gamma_{\max }}{2(\beta+1)-\gamma_{\max }}\right\}>0$. 


\subsection{Oligopoly Analysis}

Proof of Lemma 4. By (3) and (13),

$\Phi=\sum_{m=0}^{n-1} \mathrm{P}_{n-1}^{m}(\gamma(n-m)-\gamma(n-m-1))=\gamma(1) \sum_{m=0}^{n-1} \mathrm{P}_{n-1}^{m} \beta^{n-m-1}=$ $\gamma(1) \sum_{m=0}^{n-1}\left\{\sum_{M \subset M_{m}}\left[\prod_{j \in M} \lambda\left(p_{j}\right) \prod_{k \in N_{-i} \backslash M}\left(1-\lambda\left(p_{k}\right)\right)\right] \beta^{n-m-1}\right\}$.

For a given $m,\left|N_{-i} \backslash M\right|=n-m-1$, so we can re-write $\Phi$ as

$\gamma(1) \sum_{m=0}^{n-1}\left\{\sum_{M \subset M_{m}} \prod_{j \in M} \lambda\left(p_{j}\right) \prod_{k \in N_{-i} \backslash M}\left[\left(1-\lambda\left(p_{k}\right)\right) \beta\right]\right\}$.

As we first sum over all values of $m$ (for $0 \leq m \leq n-1$ ), and then over all subsets of cardinality $m$ in $\wp\left(N_{-i}\right)$, we are effectively summing up over all the subsets $M$ in $\wp\left(N_{-i}\right)$. Then, $\Phi$ becomes $\gamma(1) \sum_{M \subset \wp\left(N_{-i}\right)} \prod_{j \in M} \lambda\left(p_{j}\right) \prod_{k \in N_{-i} \backslash M}\left[\left(1-\lambda\left(p_{k}\right)\right) \beta\right]$.

Consider rival $l$ 's price $p_{l}$. Each term in the sum above contains either $\lambda\left(p_{l}\right)$ or $\left(1-\lambda\left(p_{l}\right)\right)$. In fact, for each term in the summation which includes $\lambda\left(p_{l}\right)$, there is a 'pair' which includes $\left(1-\lambda\left(p_{l}\right)\right)$ and all other multipliers are the same. Formally, take some set $M^{l} \subset \wp\left(N_{-i}\right)$ such that $l \in M^{l}$. Then, $\exists M^{-l}$ such that $M^{l}=M^{-l} \cup\{l\}$. Pairing all such subsets, we can re-write the sum above by factoring out the term $\left[\lambda\left(p_{l}\right)+\left(1-\lambda\left(p_{l}\right)\right) \beta\right]$. So,

$$
\Phi=\gamma(1)\left[\lambda\left(p_{l}\right)+\left(1-\lambda\left(p_{l}\right)\right) \beta\right] \sum_{M \subset \wp\left(N_{-i,-l}\right)} \prod_{j \in M} \lambda\left(p_{j}\right) \prod_{k \in N_{-i} \backslash M}\left[\left(1-\lambda\left(p_{k}\right)\right) \beta\right],
$$

where $\wp\left(N_{-i,-l}\right)$ is the power set of $N \backslash\{i, l\} \equiv N_{-i,-l}$. By iteration,

$$
\Phi=\gamma(1) \prod_{j \neq i}\left[\lambda\left(p_{j}\right)+\left(1-\lambda\left(p_{j}\right)\right) \beta\right]=\gamma(1) \prod_{j \neq i}\left[1+\left(1-\lambda\left(p_{j}\right)\right)(\beta-1)\right] .
$$

Proof of Proposition 2. Using (14), let

$$
\Delta(p) \equiv-\frac{n-1}{n} \prod_{j \neq i} \int_{p}^{1} \Lambda\left(p_{j}\right) d F\left(p_{j}\right)+\frac{1}{n} \prod_{j \neq i} \int_{p_{0}}^{p} \Lambda\left(p_{j}\right) d F\left(p_{j}\right)=\frac{\pi_{i}(p, k)-\pi_{i}(p, k)}{p \gamma(1)} .
$$

As $\Lambda\left(p_{j}\right)>0$ (see Lemma 4 ), it is easy to check that $\Delta\left(p_{0}\right)<0$ and $\Delta(1)>0$. Furthermore, $\Delta(p)$ is strictly increasing in $p$. As changes in $p$ only affect the integration ranges in $\Delta(p)$,

$$
\begin{aligned}
& \left.\frac{d \Delta(p)}{d p}=[1+(1-\lambda(p))(\beta-1))\right]\left(\frac{n-1}{n} \Omega_{1}+\frac{1}{n} \Omega_{2}\right) \text { where } \\
& \left.\Omega_{1}=\sum_{j \neq i}\left\{\prod_{k \neq i, k \neq j} \int_{p}^{1}\left[1+\left(1-\lambda\left(p_{j_{k}}\right)\right)(\beta-1)\right)\right] d F\left(p_{j_{k}}\right)\right\}>0 \text { and } \\
& \left.\Omega_{2}=\sum_{j \neq i}\left\{\prod_{k \neq i, k \neq j} \int_{p_{0}}^{p}\left[1+\left(1-\lambda\left(p_{j_{k}}\right)\right)(\beta-1)\right)\right] d F\left(p_{j_{k}}\right)\right\}>0 .
\end{aligned}
$$

So, to maximize its expected profit, a firm $i$ chooses according to (8), where the threshold price $\hat{p} \in\left(p_{0}, 1\right]$ satisfies $\Delta(\hat{p})=0$. 
Proof of Proposition 3. Step 1: Expected Profits. At price $p \in\left[p_{0}, \hat{p}\right)$, which is associated with $\underline{k}$, firm $i$ 's expected profit is

$$
\begin{aligned}
& \pi(p, \underline{k})= \\
& \quad p\left\{\sum_{m=0}^{n-1} C_{n-1}^{m}(1-\lambda)^{n-1-m}\left[(\lambda-F(p))^{m}(1-\gamma(n-m-1))+\frac{1}{n} \lambda^{m} \gamma(n-m-1)\right]\right\} .
\end{aligned}
$$

When $n-1-m$ competitors choose $\bar{k}$ (and so price above $\hat{p} \geq p$ ) and $m$ competitors choose $\underline{k}$ (which happens with probability $\left.\lambda^{m}(1-\lambda)^{n-1-m}\right)$ firm $i$ serves $1 / n$ of the $\gamma(n-m-1)$ confused consumers. It also serves $(1-\gamma(n-m-1))$ experts whenever all $m$ firms choosing $\underline{k}$ offer prices higher than $p$ (which happens with probability $(\lambda-F(p))^{m}(1-\lambda)^{n-1-m}$ ). This gives the term in square brackets. The term in curly brackets considers all possible combinations of $n-1$ taken $m$ and gives firm $i$ 's market share at price $p$.

Then, firm $i$ 's expected profits at $p=p_{0}$ and when $p \rightarrow \hat{p}$ are given below.

$$
\begin{array}{r}
\pi\left(p_{0}, \underline{k}\right)=p_{0}\left\{\sum_{m=0}^{n-1} C_{n-1}^{m} \lambda^{m}(1-\lambda)^{n-1-m}\left[(1-\gamma(n-m-1))+\frac{1}{n} \gamma(n-m-1)\right]\right\} \\
\lim _{p \rightarrow \hat{p}} \pi(\hat{p}, \underline{k})=\hat{p}\left[(1-\lambda)^{n-1} \varphi(1)+\frac{1}{n} \sum_{m=0}^{n-1} C_{n-1}^{m} \lambda^{m}(1-\lambda)^{n-1-m} \gamma(n-m-1)\right]
\end{array}
$$

At price $p \in[\hat{p}, 1]$, which is associated with $\bar{k}$, firm $i$ 's expected profit is

$$
\pi(p, \bar{k})=p\left[(1-F(p))^{n-1}(1-\gamma(n))+\sum_{m=0}^{n-1} C_{n-1}^{m} \lambda^{m}(1-\lambda)^{n-1-m} \frac{\gamma(n-m)}{n}\right] .
$$

When $m$ competitors choose $\underline{k}$ (and so offer prices below $\hat{p}<p$ ) and $n-1-m$ competitors choose $\bar{k}$, firm $i$ serves a share $1 / n$ of the $\gamma(n-m)$ confused consumers. The second term in square brackets considers all possible combinations of $n-1$ taken $m$. Firm $i$ serves expert consumers only if all rivals choose high complexity (that is, if $m=0$ ) and if it offers the lowest price, as reflected by the first term in square brackets.

Then, firm $i$ 's expected profit at $p=\hat{p}$ is

$$
\pi(\hat{p}, \bar{k})=\hat{p}\left[(1-\lambda)^{n-1}(1-\gamma(n))+\sum_{m=0}^{n-1} C_{n-1}^{m} \lambda^{m}(1-\lambda)^{n-1-m} \frac{\gamma(n-m)}{n}\right] .
$$

Step 2: Lambda. The equilibrium probability of choosing $\bar{k}$ presented in (15) follows from, $\lim _{p \rightarrow \hat{p}} \pi(\hat{p}, \underline{k})=\pi(\hat{p}, \bar{k}) \Leftrightarrow$ $(1-\lambda)^{n-1}(\gamma(n)-\gamma(n-1))=\frac{1}{n} \sum_{m=0}^{n-1} C_{n-1}^{m} \lambda^{m}(1-\lambda)^{n-1-m}(\gamma(n-m)-\gamma(n-m-1)) \Leftrightarrow$ $(\gamma(n)-\gamma(n-1))(1-\lambda)^{n-1}=\frac{\gamma(1)}{n}\left(\sum_{m=0}^{n-1} C_{n-1}^{m} \lambda^{m}[(1-\lambda) \beta]^{n-1-m}\right) \Leftrightarrow$ $\gamma(1)[(1-\lambda) \beta]^{n-1}=\frac{\gamma(1)}{n}[1+(1-\lambda)(\beta-1)]^{n-1}$.

Step 3: Equilibrium Profit. Evaluating the expected profit at $p=1$ and using (3), we get (16). 


$$
\begin{aligned}
& \pi(1, \bar{k})=\sum_{m=0}^{n-1} C_{n-1}^{m} \lambda^{m}(1-\lambda)^{n-1-m} \frac{\gamma(n-m)}{n}= \\
& \frac{\gamma(n)}{n\left(\beta^{n}-1\right)} \sum_{m=0}^{n-1} C_{n-1}^{m} \lambda^{m}(1-\lambda)^{n-1-m}\left(\beta^{n-m}-1\right)= \\
& \frac{\gamma(n)}{n\left(\beta^{n}-1\right)}\left[\beta \sum_{m=0}^{n-1} C_{n-1}^{m} \lambda^{m}(1-\lambda)^{n-1-m} \beta^{n-1-m}-1\right]= \\
& \frac{\gamma(n)}{n\left(\beta^{n}-1\right)}\left\{\beta \sum_{m=0}^{n-1} C_{n-1}^{m} \lambda^{m}[(1-\lambda) \beta]^{n-1-m}-1\right\}= \\
& \frac{\gamma(n)}{n\left(\beta^{n}-1\right)}\left\{\beta[1+(1-\lambda)(\beta-1)]^{n-1}-1\right\} .
\end{aligned}
$$

Then Step 2 implies that $[1+(1-\lambda)(\beta-1)]^{n-1}=n[(1-\lambda) \beta]^{n-1}$ and (16) follows.

\section{Step 4: Equilibrium Boundary Prices and Pricing Distribution Functions.}

- $p_{0}$ and $\hat{p}$ are defined by $\pi\left(p_{0}, \underline{k}\right)=\pi$ and $\lim _{p \rightarrow \hat{p}} \pi(\hat{p}, \underline{k})=\pi$, where the LHS terms are given in (A4) and (A5), respectively, the RHS term is given by (16), and $\lambda$ follows from (15).

Firm $i$ 's market share at $p_{0}$ - the term in curly brackets in (A4) - is larger than its market share when $p \rightarrow \hat{p}$ - the term in square brackets in (A5) because $(1-\lambda)^{n-1}(1-\gamma(n-1))<$ $\sum_{m=0}^{n-1} C_{n-1}^{m} \lambda^{m}(1-\lambda)^{n-1-m}(1-\gamma(n-m-1))$. This implies that $p_{0}<\hat{p}$ as the equilibrium constant profit condition requires $\pi\left(p_{0}, \underline{k}\right)=\lim _{p \rightarrow \hat{p}} \pi(\hat{p}, \underline{k})$.

Moreover, $\hat{p} \leq 1$, with equality iff $\gamma(n)=1$. Using (16) and (A5), $\hat{p} \leq 1$ requires that

$\frac{1}{n} \sum_{m=0}^{n-1} C_{n-1}^{m} \lambda^{m}(1-\lambda)^{n-1-m} \gamma(n-m) \leq$

$(1-\lambda)^{n-1}(1-\gamma(n-1))+\frac{1}{n} \sum_{m=0}^{n-1} C_{n-1}^{m} \lambda^{m}(1-\lambda)^{n-1-m} \gamma(n-m-1) \Leftrightarrow$

$\frac{1}{n} \sum_{m=0}^{n-1} C_{n-1}^{m} \lambda^{m}(1-\lambda)^{n-1-m}(\gamma(n-m)-\gamma(n-m-1)) \leq(1-\lambda)^{n-1}(1-\gamma(n-1)) \Leftrightarrow$

$\frac{1}{n}[\lambda+(1-\lambda) \beta]^{n-1}-[(1-\lambda) \beta]^{n-1} \leq(1-\lambda)^{n-1} \frac{(1-\gamma(n))}{\gamma(1)}$.

Expression (15) implies that the LHS is equal to zero. The RHS is nonnegative and may be equal to zero only if $\gamma(n)=1$. So $\hat{p}<1$ if $\gamma(n)<1$ and $\hat{p}=1$ if $\gamma(n)=1$.

Below, we focus on the pricing c.d.f.s. Note that $\pi$ is defined in (16).

- For $p \in\left[p_{0}, \hat{p}\right), F(p)$ is implicitly determined by $\pi(\underline{k}, p)=\pi$.

Differentiating w.r.t. $p$ both sides gives

$-F^{\prime}(p) \sum_{m=1}^{n-1} C_{n-1}^{m}\left[m(\lambda-F(p))^{m-1}(1-\lambda)^{n-1-m}(1-\gamma(n-m-1))\right]=-\frac{\pi}{p^{2}}$.

Here, as $F(p)<F(\hat{p})=\lambda$, the summation is strictly positive. Then, $F(p)$ is strictly increasing in $p$.

For $p \in[\hat{p}, 1], F(p)$ is implicitly determined by $\pi(\bar{k}, p)=\pi \Leftrightarrow$

$(1-F(p))^{n-1}(1-\gamma(n))+\sum_{m=0}^{n-1} C_{n-1}^{m} \lambda^{m}(1-\lambda)^{n-1-m} \frac{\gamma(n-m)}{n}=\frac{\pi}{p}$.

It is clear that $F(p)$ is strictly increasing on this interval. Moreover, $F(p)$ is continuous at $\hat{p}$, $F\left(p_{0}\right)=0$, and $F(1)=1$.

\section{Step 5: No Profitable Unilateral Deviations.}

Consider a unilateral deviation to $\left(p<p_{0}, \underline{k}\right)$. The deviator's market share is equal to that corresponding to $p=p_{0}$ but, as the price is lower, this deviation is not profitable. 
Consider a unilateral deviation to $(p>\hat{p}, \underline{k})$. This results in deviation profit $\pi^{d}(p, \underline{k})=p\left[(1-F(p))^{n-1}(1-\gamma(n-1))+\frac{1}{n} \sum_{m=0}^{n-1} C_{n-1}^{m}(1-\lambda)^{n-1-m} \lambda^{m} \gamma(n-m-1)\right]$.

But $\pi^{d}(p, \underline{k})<\pi(p, \bar{k}) \Leftrightarrow$ $(1-F(p))^{n-1}(1-\gamma(n-1))+\frac{1}{n} \sum_{m=0}^{n-1} C_{n-1}^{m}(1-\lambda)^{n-1-m} \lambda^{m} \gamma(n-m-1)<$ $(1-F(p))^{n-1}(1-\gamma(n))+\frac{1}{n} \sum_{m=0}^{n-1} C_{n-1}^{m} \lambda^{m}(1-\lambda)^{n-1-m} \gamma(n-m) \Leftrightarrow$ $(1-F(p))^{n-1}(\gamma(n)-\gamma(n-1))<\frac{1}{n} \sum_{m=0}^{n-1} C_{n-1}^{m} \lambda^{m}(1-\lambda)^{n-1-m}(\gamma(n-m)-\gamma(n-m-1)) \Leftrightarrow$ $(1-F(p))^{n-1} \beta^{n-1}<\frac{1}{n} \sum_{m=0}^{n-1} C_{n-1}^{m} \lambda^{m}[(1-\lambda) \beta]^{n-1-m} \Leftrightarrow$ $(1-F(p))^{n-1} \beta^{n-1}<\frac{1}{n}[\lambda+(1-\lambda) \beta]^{n-1}$.

The last inequality follows from the fact that, as $p>\hat{p}$, $(1-F(p))^{n-1} \beta^{n-1}<(1-F(\hat{p}))^{n-1} \beta^{n-1}=(1-\lambda)^{n-1} \beta^{n-1}$.

It is then easy to check using (15) that $(1-\lambda)^{n-1} \beta^{n-1}=\frac{[\lambda+(1-\lambda) \beta]^{n-1}}{n}$.

Consider a unilateral deviation to $(p<\hat{p}, \bar{k})$. This results in deviation profit.

$\pi^{d}(p, \bar{k})=p\left\{\sum_{m=0}^{n-1} C_{n-1}^{m}(1-\lambda)^{n-1-m}\left[(\lambda-F(p))^{m}(1-\gamma(n-m))+\lambda^{m} \frac{\gamma(n-m)}{n}\right]\right\}$.

But, $\pi^{d}(p, \bar{k})<\pi(p, \underline{k})$ as

$\sum_{m=0}^{n-1} C_{n-1}^{m}(1-\lambda)^{n-1-m}\left[(\lambda-F(p))^{m}(1-\gamma(n-m))+\lambda^{m} \frac{\gamma(n-m)}{n}\right]<$

$\sum_{m=0}^{n-1} C_{n-1}^{m}(1-\lambda)^{n-1-m}\left[(\lambda-F(p))^{m}(1-\gamma(n-m-1))+\lambda^{m} \frac{\gamma(n-m-1)}{n}\right] \Leftrightarrow$

$\sum_{m=0}^{n-1} C_{n-1}^{m}(1-\lambda)^{n-1-m}(\gamma(n-m)-\gamma(n-m-1))\left[-(\lambda-F(p))^{m}+\frac{\lambda^{m}}{n}\right]<0 \Leftrightarrow$

$\sum_{m=0}^{n-1} C_{n-1}^{m}[(1-\lambda) \beta]^{n-1-m}\left[-(\lambda-F(p))^{m}+\frac{\lambda^{m}}{n}\right]<0 \Leftrightarrow$

$-\sum_{m=0}^{n-1} C_{n-1}^{m}[(1-\lambda) \beta]^{n-1-m}(\lambda-F(p))^{m}+\frac{1}{n} \sum_{m=0}^{n-1} C_{n-1}^{m}[(1-\lambda) \beta]^{n-1-m} \lambda^{m}<0 \Leftrightarrow$

$-[(1-\lambda) \beta+\lambda-F(p)]^{n-1}+\frac{1}{n}[(1-\lambda) \beta+\lambda]^{n-1}<0$.

The last inequality follows as for $p<\hat{p}$,

$-[(1-\lambda) \beta+\lambda-F(p)]^{n-1}<-[(1-\lambda) \beta]^{n-1}$.

But, using (15), $-[(1-\lambda) \beta]^{n-1}+\frac{1}{n}[(1-\lambda) \beta+\lambda]^{n-1}=0$.

\subsection{Discussion and Policy Implications}

\section{Proof of Corollary 3.}

For $\beta \neq 1, \lambda$ is defined in (15) and for $\beta=1$ it is defined in (17). It is easy to see that when evaluated at $\beta=1$, expression (15) reduces to (17). Therefore, this proof uses (15) to cover both 
cases.

$\operatorname{Re}$-write $(15)$ as $\lambda(\tilde{n})=1-\frac{\tilde{n}}{\beta-(\beta-1) \tilde{n}}$ where $\tilde{n}=n^{-1 /(n-1)} \in(0,1)$,

with $\frac{d \tilde{n}}{d n}=\frac{n^{-n /(n-1)}(1-n+n \log n)}{(n-1)^{2}}>0$.

Using the chain rule, $\frac{d \lambda}{d n}=\frac{d \lambda}{d \tilde{n}} \frac{d \tilde{n}}{d n}<0$ as $\frac{d \lambda}{d \tilde{n}}=-\frac{\beta}{[\beta-(\beta-1) \tilde{n}]^{2}}<0$.

$\lim _{n \rightarrow \infty} \tilde{n}=\exp \left[\lim _{n \rightarrow \infty} \ln \left(n^{-1 /(n-1)}\right)\right]=$

$\exp \left[\lim _{n \rightarrow \infty}\left(-\frac{\ln n}{n-1}\right)\right]=\exp \left[-\lim _{n \rightarrow \infty}\left(\frac{\ln n}{n-1}\right)\right]$.

By L'Hôpital's rule, $\lim _{n \rightarrow \infty}\left(\frac{\ln n}{n-1}\right)=\lim _{n \rightarrow \infty} \frac{1}{n}=0$. So, $\lim _{n \rightarrow \infty} \tilde{n}=1$ and $\lim _{n \rightarrow \infty} \lambda=0$.

Moreover, $\frac{d \lambda}{d \gamma(n)}=0$ and $\frac{d \lambda}{d \beta}=\frac{\tilde{n}(1-\tilde{n})}{[\beta-(\beta-1) \tilde{n}]^{2}}>0$.

\section{Proof of Corollary 4.}

Suppose $\beta \in(0,1) . \lim _{n \rightarrow \infty} \exp [\gamma]=\lim _{n \rightarrow \infty} \frac{\gamma_{\max }\left\{1-[1+(1-\lambda)(\beta-1)]^{n}\right\}}{1-\beta^{n}}$.

In this case, $\beta<1$ and $1+(1-\lambda)(\beta-1)<1$.

As $\lim _{n \rightarrow \infty}\left(1-\beta^{n}\right)=0$ and $\lim _{n \rightarrow \infty}\left\{1-[1+(1-\lambda)(\beta-1)]^{n}\right\}=1$, it follows that $\lim _{n \rightarrow \infty} \exp [\gamma]=\gamma_{\max }$

- Suppose $\beta>1$. It is convenient to write

$\lim _{n \rightarrow \infty} \exp [\gamma]=\lim _{n \rightarrow \infty} \frac{\gamma_{\max }\left\{[\beta-\lambda(\beta-1)]^{n}-1\right\}}{\beta^{n}-1}=$

$\gamma_{\max } \frac{\lim _{n \rightarrow \infty}\left\{[\beta-\lambda(\beta-1)]^{n}-1\right\}}{\lim _{n \rightarrow \infty}\left(\beta^{n}-1\right)}$.

$\lim _{n \rightarrow \infty}\left\{[\beta-\lambda(\beta-1)]^{n}-1\right\}=\lim _{n \rightarrow \infty}\left(\beta^{n}-1\right)=\infty$, as $\beta>\beta-\lambda(\beta-1)=1+(1-\lambda)(\beta-1)>$ 1 .

But the first inequality implies that the denominator converges faster. Then $\lim _{n \rightarrow \infty} \exp [\gamma]=0$.

Suppose $\beta=1$. By Corollary $3, \lim _{n \rightarrow \infty}(1-\lambda)=1$. Then, $\lim _{n \rightarrow \infty} \gamma(n)(1-\lambda)=\gamma_{\max }$.

In all cases, the impact of $\gamma_{\max }$ is straightforward.

Example 3: Expected share of confused consumers as a function of $n$.

$$
E[\gamma]=\left\{\begin{array}{c}
\frac{\left[1-n^{-1 /(n-1)} /\left(1+n^{-1 /(n-1)}\right)\right]^{n}-1}{(1 / 2)^{n}-1} \text { for } \beta=1 / 2 \\
n^{-1 /(n-1)} \text { for } \beta=1 \\
\frac{\left[2 /\left(2-n^{-1 /(n-1)}\right)\right]^{n}-1}{2^{n}-1} \text { for } \beta=2
\end{array}\right.
$$

\section{Proof of Corollary 5.}

- Consider $\beta \neq 1$. Using (15) and (16), industry profit can be written as 
$\pi_{n}=\frac{\gamma_{\max }\left\{\frac{\beta^{n}}{\left[1+(\beta-1)\left(1-n^{-1 /(n-1)}\right)\right]^{n-1}}-1\right\}}{\beta^{n}-1}=$

$\frac{\gamma_{\max }\left[\beta-(\beta-1) n^{-1 /(n-1)}\right]\left[\frac{\beta}{\beta-(\beta-1) n^{-1 /(n-1)}}\right]^{n}-1}{\beta^{n}-1}=$

$\frac{\gamma_{\max }\left[\beta-(\beta-1) n^{-1 /(n-1)}\right][\lambda+(1-\lambda) \beta]^{n}-1}{\beta^{n}-1}$.

As $\lim _{n \rightarrow \infty}\left[\beta-(\beta-1) n^{-1 /(n-1)}\right]=1$, it follows that

$\lim _{n \rightarrow \infty} \pi_{n}=\lim _{n \rightarrow \infty} \frac{\gamma_{\max }[\lambda+(1-\lambda) \beta]^{n}-1}{\beta^{n}-1}$.

But, the RHS is $\lim _{n \rightarrow \infty} \exp [\gamma]$. Using Corollary 4, the results follow.

Using (16) and (19),

$\pi_{n}-\exp [\gamma]=\gamma_{\max }\left\{\frac{n(1-\lambda)^{n-1} \beta^{n}-1}{\beta^{n}-1}-\frac{[1+(1-\lambda)(\beta-1)]^{n}-1}{\beta^{n}-1}\right\}=$

$\gamma_{\max } \frac{n(1-\lambda)^{n-1} \beta^{n}-[1+(1-\lambda)(\beta-1)]^{n}}{\beta^{n}-1}=$

$\gamma_{\max } \frac{\beta[1+(1-\lambda)(\beta-1)]^{n-1}-[1+(1-\lambda)(\beta-1)]^{n}}{\beta^{n}-1}$,

where the last equality uses step3 in the proof of Proposition 3.

Then, $\pi_{n}-\exp [\gamma]=\gamma_{\max } \frac{[1+(1-\lambda)(\beta-1)]^{n-1}(\beta-1)(2-\lambda)}{\beta^{n}-1}>0$.

- Consider $\beta=1$, using (17) and (18), $\lim _{n \rightarrow \infty} \pi_{n}=\lim _{n \rightarrow \infty}\left\{\gamma_{\max }\left[\frac{\lambda}{n}+(1-\lambda)\right]\right\}$. Using

Corollary 3 , the convergence result follows.

Using (18) and (19),

$\pi_{n}-\exp [\gamma]=\gamma_{\max }\left[\frac{\lambda+n(1-\lambda)}{n}-(1-\lambda)\right]=\frac{\gamma_{\max } \lambda}{n}>0$.

\section{References}

Baye, M., J. Morgan, and P. Scholten (2006): "Information, Search, and Price Dispersion," in Handbook of Economics and Information Systems, ed. by T. Hendershott. Elsevier Press, Amsterdam.

Campbell, J. Y. (2016): "Richard T. Ely Lecture Restoring Rational Choice: The Challenge of Consumer Financial Regulation," American Economic Review, 106(5), 1-30.

CARLin, B. (2009): "Strategic Price Complexity in Retail Financial Markets," Journal of Financial Economics, 91(3), 278-287.

Célérier, C., And B. Vallée (2017): "Catering to Investors through Security Design: Headline Rate and Complexity," The Quarterly Journal of Economics, 132(3), 1469-1508.

Chioveanu, I. (2018): "An Asymmetric Duopoly Model of Price Framing," BE Journal of Theoretical Economics, 19(2). 
(2019): "Prominence, Complexity, and Pricing," International Journal of Industrial Organization, 63, 551-582.

Chioveanu, I., and J. Zhou (2013): "Price Competition with Consumer Confusion," Management Science, 59(11), 2450-2469.

Competition and Markets Authority (2015): "Retail Banking Market Investigation: Summary of Provisional Findings Report," available at https://www.gov.uk/cma-cases/review-ofbanking-for-small-and-medium-sized-businesses-smes-in-the-uk.

De Roos, N. (2018): "Collusion with Limited Product Comparability," The RAND Journal of Economics, 49(3), 481-503.

European Commission (2007): "Staff Working Document Accompanying the White Paper on the Integration of EU Mortgage Credit Markets," available at http://eur-lex.europa.eu.

Ghent, A. C., W. N. Torous, and R. I. Valkanov (2017): "Complexity in Structured Finance," The Review of Economic Studies, 86(2), 694-722.

Gu, Y., And T. Wenzel (2014): "Strategic Obfuscation and Consumer Protection Policy," Journal of Industrial Economics, 62(4), 632-660.

Hortaçsu, A., S. A. Madanizadeh, and S. L. Puller (2017): "Power to choose? An analysis of consumer inertia in the residential electricity market," American Economic Journal: Economic Policy, 9(4), 192-226.

Independent Banking Commission (2011): "Final Report Recommendations," available at http://bankingcommission.independent.gov.uk/.

Piccione, M., and R. Spiegler (2012): "Price Competition under Limited Comparability," Quarterly Journal of Economics, 127(1), 97-135.

RosenthaL, R. (1980): "A Model in which an Increase in the Number of Sellers Leads to a Higher Price," Econometrica, 48(6), 1575-1579.

Spiegler, R. (2016): "Choice Complexity and Market Competition," Annual Review of Economics, 8, 1-25.

Varian, H. (1980): "A Model of Sales," American Economic Review, 70(4), 651-659.

Vives, X. (2001): Oligopoly Prcing: Old Ideas and New Tools. The MIT Press.

Woodward, S., and R. Hall (2012): "Diagnosing Consumer Confusion and Sub-optimal Shopping Effort: Theory and Mortgage-Market Evidence," American Economic Review, 102(7), $3249-3276$. 